1 Persistent fibrosis and decreased cardiac function following cardiac injury in the

2 Ctenopharyngodon idella (grass carp)

3 Daniel W Long ${ }^{1}$, Charles H Webb IV ${ }^{1}$, Yadong Wang ${ }^{1 *}$

$4{ }^{1}$ Meinig School of Biomedical Engineering, Cornell University, Ithaca, NY, USA

5

6 Short title: Cardiac fibrosis in grass carp

7

$8 \quad{ }^{*}$ Corresponding author:

9 Yadong Wang, Ph.D.

10 Meinig School of Biomedical Engineering

11 Cornell University

$12 \quad 279$ Kimball Hall

13134 Hollister Drive

14 Tel: $1-412-624-7196$

15 Email: yw839@cornell.edu

16

17 Keywords: Cyprinid, cardiac fibrosis, cardiac function, heart repair 


\section{Summary statement}

19 Grass carp, a member of the Cyprinidae family that includes regenerative zebrafish, do

20 not regenerate functional cardiac tissue after cryoinjury. Instead, healing progresses

21 through collagen deposition and scar formation. 


\section{Abstract}

23 Following the discovery of heart regeneration in zebrafish, several more species within

24 the Cyprinidae family have been found to have the same capability, suggesting heart

25 regeneration may be conserved within this family. Although gonad regeneration has

26 been observed in grass carp (Ctenopharyngodon idella), one of the largest cyprinid fish,

27 the species' response to cardiac injury has not been characterized. Surprisingly, we

28 found cardiomyocytes do not repopulate the injured region following cryoinjury to the

29 ventricle, instead exhibiting unresolved fibrosis and decreased cardiac function that

30 persists for the 8-week duration of this study. Compared to other cyprinid fish studied,

31 infiltration of macrophages is delayed and muted in this model. Additionally, fibroblasts

32 are depleted following injury, a phenomenon not previously described in any cardiac

33 model. This study shows that heart regeneration is not conserved among the Cyprinidae

34 family and suggests the important role of non-fibroblasts in chronic fibrosis. Further

35 study of these phenomenon may reveal the underlying differences between

36 regeneration versus unresolved fibrosis in heart disease. 


\section{Introduction}

38 Organ regeneration is a phenomenon that occurs in select species across the animal kingdom (Lenhoff and Lenhoff, 1986; Morgan, 1901). Humans are generally incapable of regenerating damaged tissues with a few notable exceptions (Michalopoulos, 2007). Heart regeneration specifically is not seen in humans but is reported in several adult non-mammalian species and neonatal mammals (Cano-Martínez et al., 2010; Liao et al., 2017; Porrello et al., 2011; Poss et al., 2002; Ye et al., 2018). Following the discovery of cardiac regeneration in zebrafish after ventricle resection in 2002 (Poss et al., 2002), several varieties of fish have been investigated for their regenerative potential after cardiac injury. To date, cardiac regeneration has been observed in several cyprinid fish, including the zebrafish, giant danio, and goldfish, along with a surface variety of Astyanax mexicanus outside the Cyprinidae family (Grivas et al., 2014; Lafontant et al., 2012a; Poss et al., 2002; Stockdale et al., 2018). In contrast, the cave-dwelling Pachón variety of Astyanax mexicanus and medaka (Oryzias latipes) do not exhibit signs of cardiac regeneration following injury, neither of which are in the Cyprinidae family (Fig. 1A) (Ito et al., 2014; Stockdale et al., 2018).

While cardiomyocyte proliferation was thought to be the main driver of cardiac regeneration in fish, the recent discovery of widespread cardiomyocyte proliferation in non-regenerative Pachón Axtyanax mexicanus indicates that there are alternative regulators of cardiac injury at play (Stockdale et al., 2018). However, with only two nonregenerative fish species currently reported, it is difficult to assess which mechanisms are essential regulators of regeneration versus chronic fibrosis. Therefore, research on responses to cardiac injury in additional fish species will inform what cellular behaviors are chiefly responsible for driving cardiac regeneration versus chronic fibrosis. Grass carp, or Ctenopharyngodon idella, are an Asian variety of carp within the Cyprinidae family of fish (Fig. 1A). Unlike previously reported zebrafish (<1 g) (GómezRequeni et al., 2010), giant danio (1.6 g) (Lafontant et al., 2012b), medaka (<2 g) (Ding et al., 2010; Lafontant et al., 2012a), and goldfish (3.4 g) (Grivas et al., 2014) models of cardiac injury, grass carp used in this study are over $400 \mathrm{~g}$ and over $30 \mathrm{~cm}$ in length (Fig. 1B). These fish are capable of exceeding $25 \mathrm{~kg}$ in mass and 1 meter in length in aquaculture conditions, thus their size is several orders-of-magnitude greater than the 
68 previously studied species (Stich et al., 2013). As an invasive species to the United

69 States, early efforts to sterilize these fish by removal of the gonads were ineffective due

70 to their ability to regenerate reproductive organs within months of removal (Underwood

71 et al., 1986). The regenerative potential of other organs in this fish have not been further

72 explored. In fact, the regenerative potential of cardiac tissue in any large fish species is

73 unknown.

74 In the present study we design a method to reproducibly injure the heart of the grass

75 carp, with a survival rate $>95 \% 24$ hours post-surgery. We investigated the cellular and

76 functional response of the heart following injury. Our study provides a surgical model for

77 investigation of cardiac injury in large fish species and suggests that grass carp are the

78 first reported cyprinids that exhibit chronic fibrosis rather than functional regeneration.

79 Additionally, fibroblast and macrophage responses to injury are different from reported

80 cyprinid fish species and warrant further investigation. 


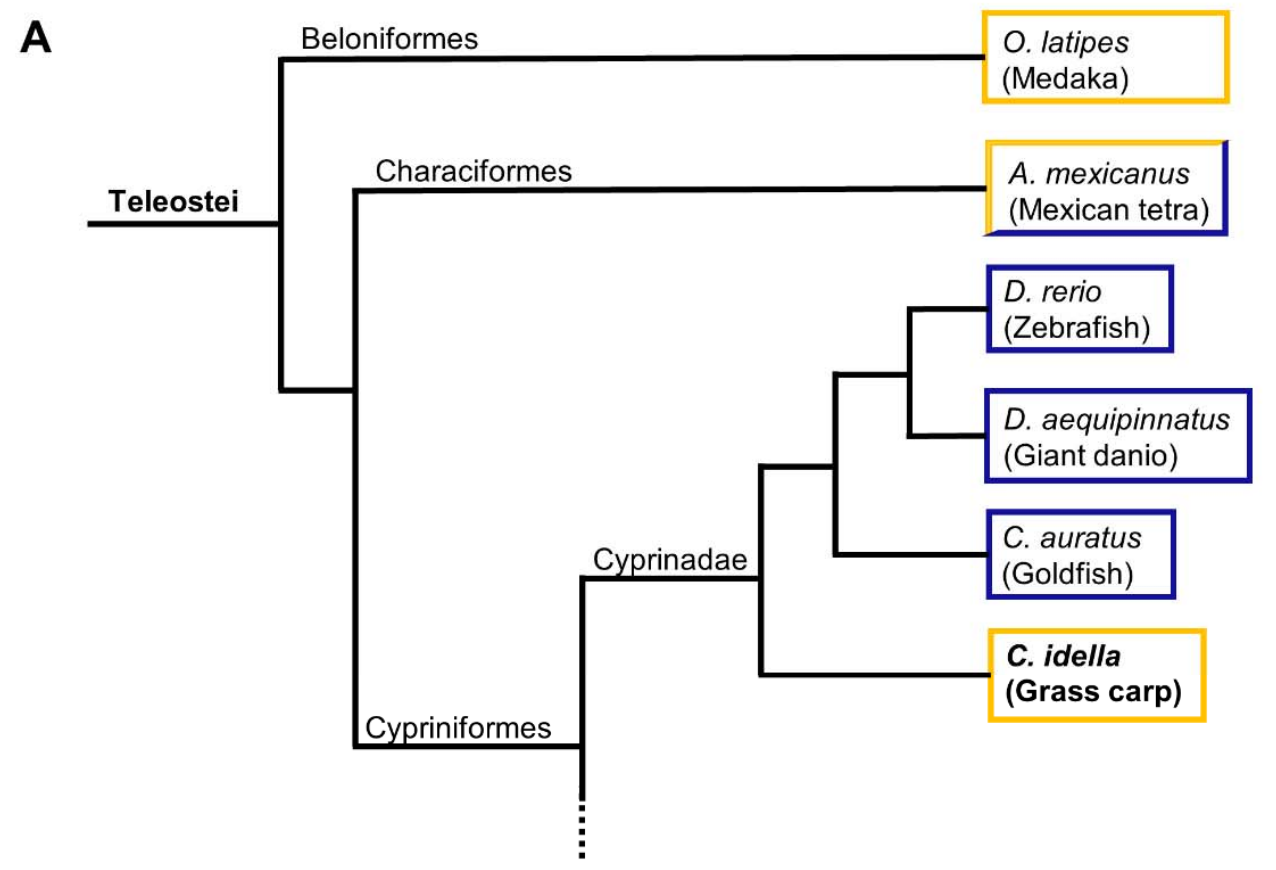

B

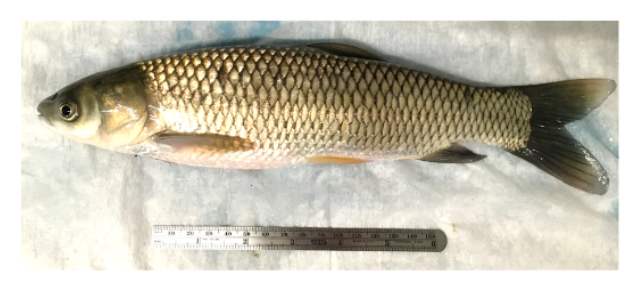

C

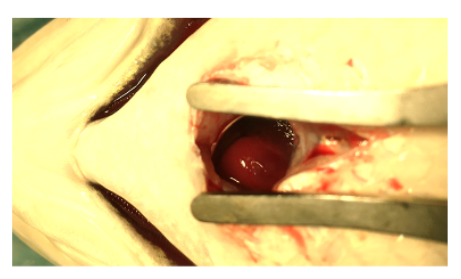

Regeneration

Fibrosis
D

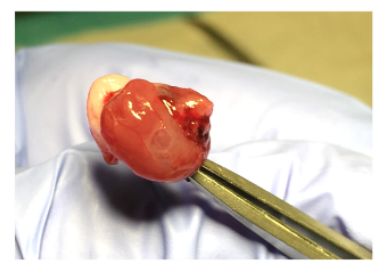

Figure 1.

Figure 1: Grass carp characterization and taxonomy

83 (A) All previously investigated cyprinids exhibit cardiac regeneration following injury with

84 exception to grass carp presented in the present study. Medaka and at least one variant

85 of $A$. mexicanus, distant relatives of the grass carp, instead heal via fibrosis following

86 cardiac injury. (B) Grass carp used in this study were approximately $300 \mathrm{~mm}$ in length

87 (total length) and $435 \pm 112 \mathrm{~g}$ in mass. (C) Alm retractors were used to separate the

88 operculum and allow direct visualization of the ventricle. The injured zone at the apex is

89 outlines in yellow. (D) Explant at 56 DPI reveals white scar tissue rather than healthy

90 pink tissue indicative of full regeneration. 


\section{Results}

92 2.1 Cardiac wound healing following cryoinjury in the grass carp

93 The use of cryoinjury was selected over resection due to limited access to the apex in

94 grass carp heart with scissors (Fig. 1C). The use of a $2 \mathrm{~mm}$ stainless steel cryoprobe

95 resulted in an injury covering $10-15 \%$ of the ventricle area at its maximum depth (Fig.

96 2B), similar to previously reported goldfish injury (Grivas et al., 2014). Although this is a

97 relatively small injury compared to standard zebrafish and giant danio models (Chablais

98 et al., 2011; Lafontant et al., 2012a), larger injuries resulting from longer freezing times

99 and larger probes resulted in greater than $85 \%$ mortality within 14 days of injury.

100 Acid fuchsin orange G (AFOG) staining of the fish ventricle was used to identify and

101 quantify the injury size over 8 weeks post-injury. Uninjured hearts contained a collagen-

102 rich bulbus arteriosus in blue and a healthy ventricle and atrium stained orange (Fig.

103 2A). Fibrin deposits and the injury site were easily visible by 1 day post injury (DPI),

104 which was converted to a collagen-rich scar by 56 DPI (Fig. 2B,C). Quantification of the

105 injury size over time revealed that although the injury does reduce in size by $14 \mathrm{DPI}$

106 relative to 1 and $4 \mathrm{DPI}$ ( $p<0.05$ and $p<0.01$, respectively), there is no further reduction

107 between 14 and 56 DPI (Fig. 2D). Additionally, a white scar region is grossly visible on

108 the ventricle $56 \mathrm{DPI}$ at explant, indicating an incomplete regeneration of the injured

109 ventricle (Fig. 1D). 


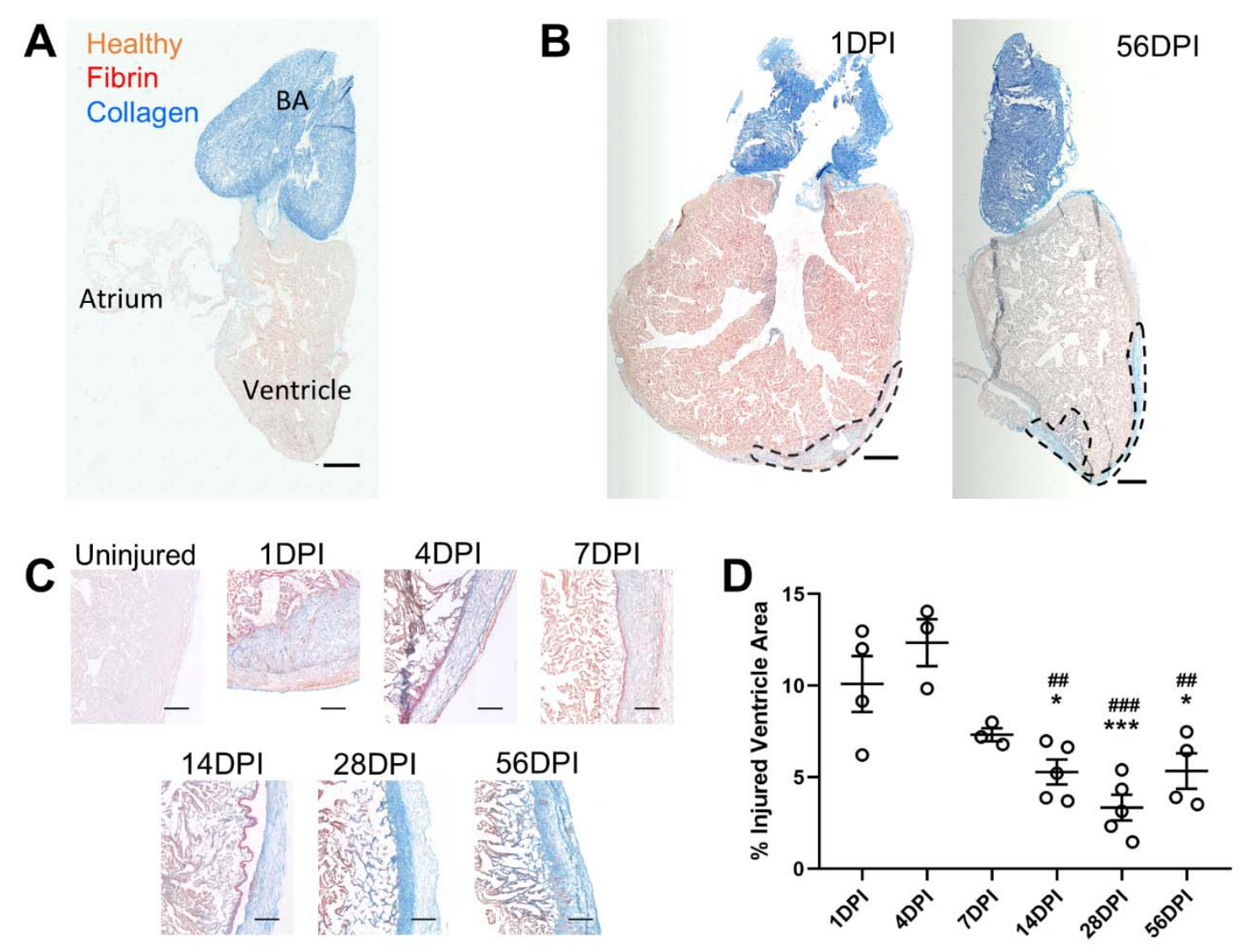

Figure 2.

112 Figure 2: Heart anatomy and injury response

113 (A) AFOG-stained grass carp hearts reveal a single atrium, a ventricle composed of a

114 cortical outer myocardium and trabecular inner myocardium, and the collagenous

115 bulbus arteriosus (BA), which serves at the outflow tract (scale bar $=1 \mathrm{~mm}$ ). (B) The

116 injury site is visible at the ventricle apex $1 \mathrm{DPI}$, which is still visible $56 \mathrm{DPI}$ as a fibrous

117 scar, outlined in black (scale bar $=1 \mathrm{~mm}$ ). (C) AFOG staining reveals the progression of

118 the injury site from a fibrin-rich wound 1-7 DPI to a collagen-rich scar by 28-56 DPI

119 (scale bar $=200 \mu \mathrm{m}$ ). (D) Quantification of the injury size reveals a significant reduction

120 from early to late-stage injuries, although injury size does not decrease further after 14

121 DPI. ( ${ }^{*} p<0.05,{ }^{* * *} p<0.001$ relative to $1 \mathrm{DPI} ;{ }^{\# \#} p<0.01$, ${ }^{\# \#} p<0.001$ relative to 4 DPI). 


\subsection{Cardiac function fails to recover following injury}

In addition to histological assessments, echocardiography-based measurements were used to assess functional regeneration of cardiac tissue. B-mode echocardiography along the long-axis was used to measure ventricle area and length at systole and diastole (Fig. 3A). Cryoinjury to the hearts resulted in an immediate decrease in fractional area change (FAC) that persisted through the rest of the 56-day study period (Fig. 3B; $p<0.001$ relative to uninjured at $4 \mathrm{DPI}, \mathrm{p}<0.05$ relative to uninjured at 1,7 , 14 , and $56 \mathrm{DPI})$. Similarly, fractional shortening (FS) values were significantly reduced by $1 \mathrm{DPI}$ and persisted through $56 \mathrm{DPI}$ (Fig. 3C; $p<0.001$ relative to uninjured at 1,4 , $7,14$, and $56 \mathrm{DPI})$. Interestingly, neither FAC nor FS values are significantly lower than uninjured fish at $28 \mathrm{DPI}$, although the scar is clearly still present (Fig. 2). This assessment paired with measurements of injury size (Fig. 2) indicates a lack of cardiac regeneration both histologically and functionally.

\subsection{Cryoinjury induces localized and acute apoptosis}

Cardiac injury, especially cryoinjury, results in high levels of apoptosis in the injury zone in other models (Chablais et al., 2011; Darehzereshki et al., 2015; Polizzotti et al., 2015; Talman and Ruskoaho, 2016). TUNEL staining of cardiac tissues in the injured zone revealed high levels of apoptosis at 1 and 4 DPI (Fig. 4A,B; $p<0.001$ relative to uninjured), which were back at baseline levels by 7 DPI. Except for a slight increase in TUNEL+ cells at $14 \mathrm{DPI}(\mathrm{p}<0.05$ relative to uninjured), apoptosis levels were low for the remainder of the study period. TUNEL staining in remote regions of the heart did not reveal a significant increase in apoptosis at any point after injury (Fig. 4A,C). Together these results indicate that cryoinjury in the grass carp heart causes an acute response of localized apoptosis that quickly resolves without spreading into otherwise healthy tissue. 

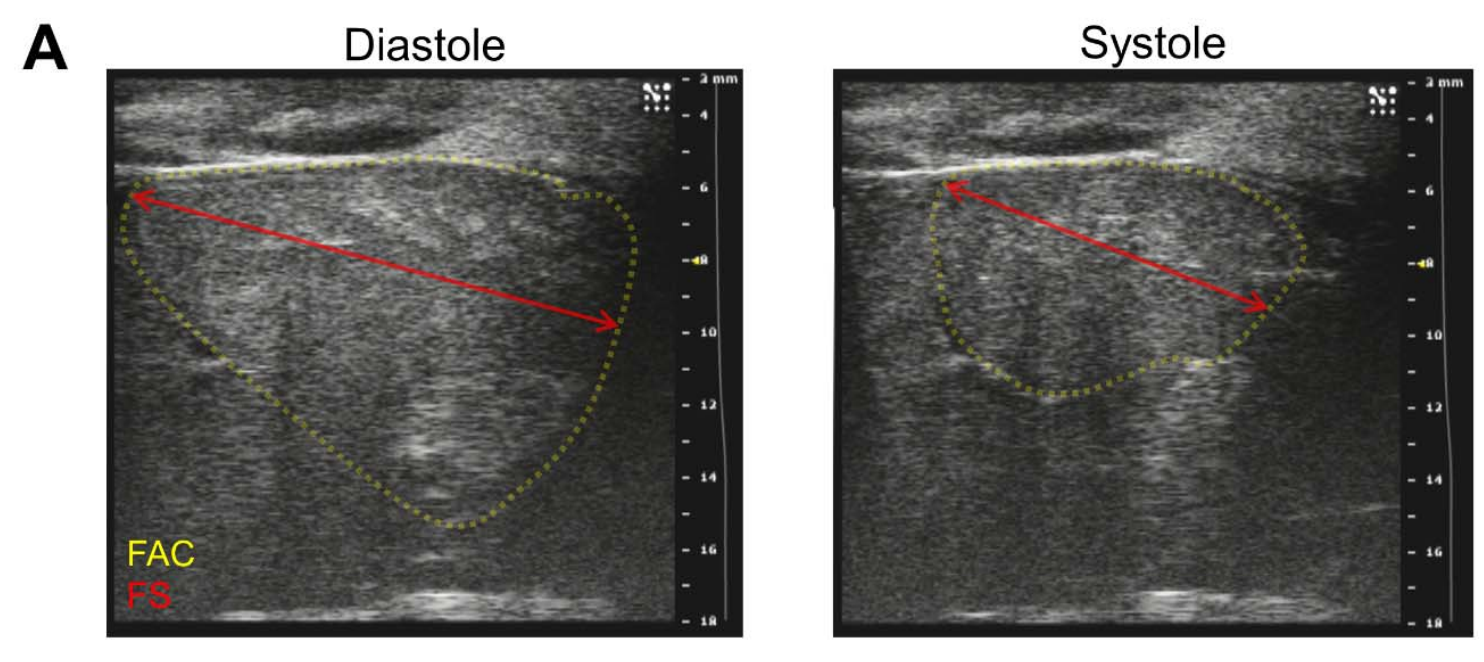

B
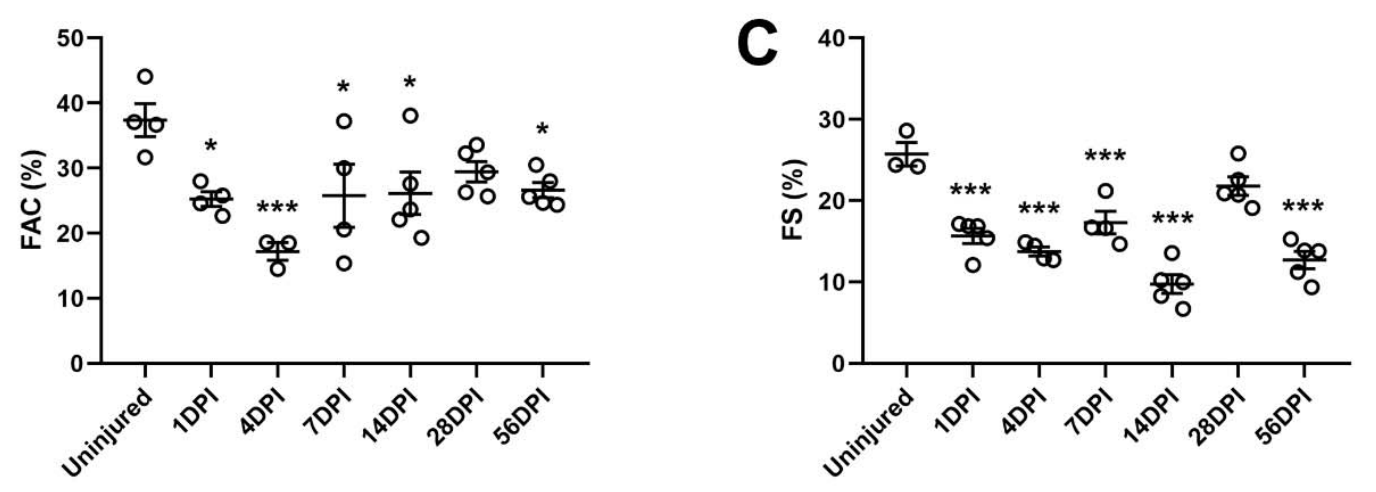

Figure 3.

150 Figure 3: Cardiac function does not recover following injury.

151 (A) Long-axis B-mode echocardiography of the heart at diastole and systole were used

152 to quantify FAC and FS measurements following injury. (B) FAC values, though they

153 improved from their minimum at $4 \mathrm{DPI}$, did not recover healthy function by $56 \mathrm{DPI}$. (C)

154 Similarly, FS values did not improve to baseline values by 56 DPI, although FS at 28

155 DPI appeared to partially recover. ( ${ }^{*} p<0.05,{ }^{* *} p<0.001$ relative to uninjured). 

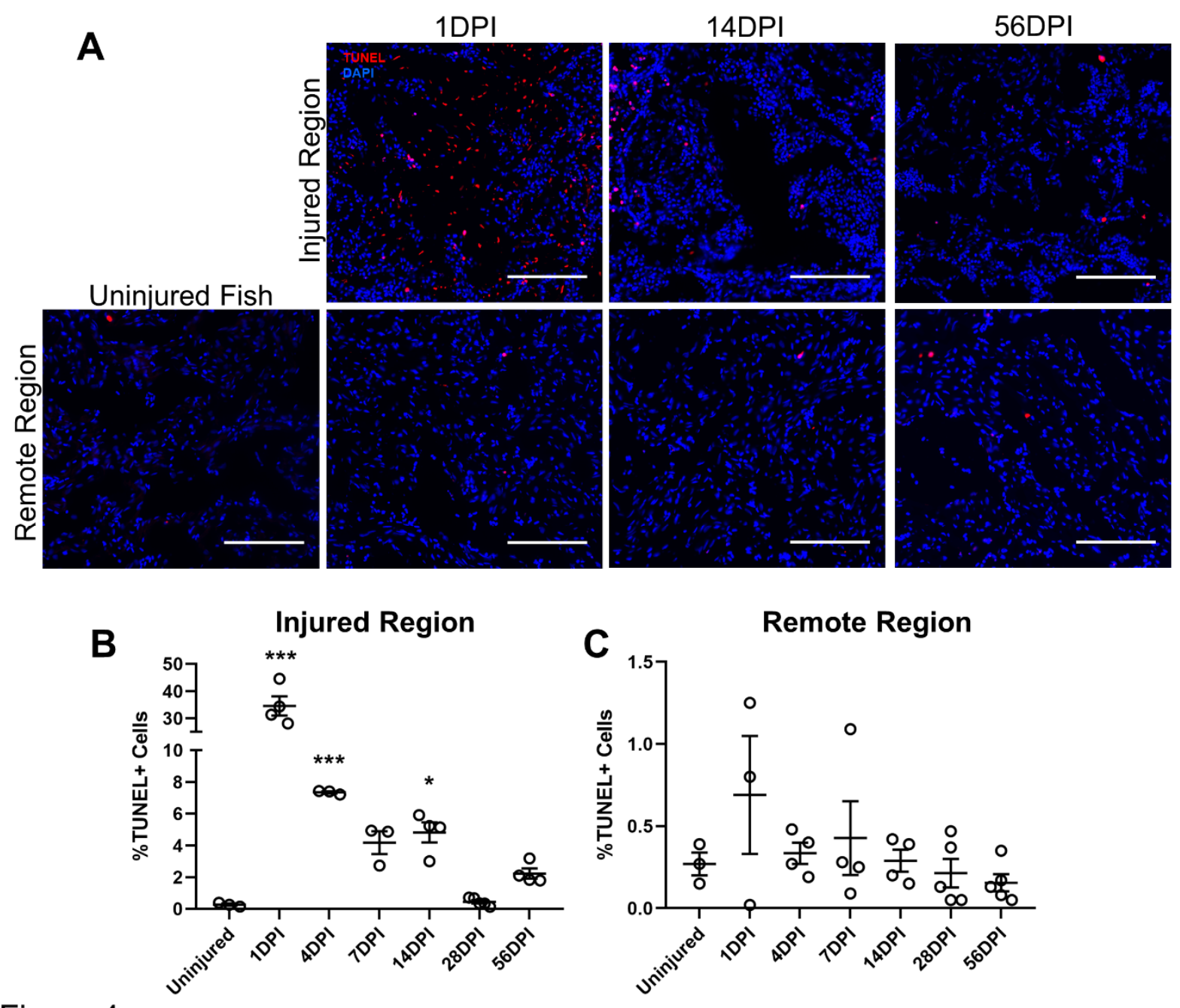

Figure 4.

Figure 4. TUNEL Staining reveals a localized burst of apoptosis in the injured region.

160 (A) Apoptotic cells are visible at all points after injury, although this is most pronounced 161 in the injured region and at early timepoints following injury. (B) Quantification of 162 TUNEL+ cells reveals high levels of apoptosis at 1, 4, and 14 DPI in the injured region.

163 (C) TUNEL+ cells are not present in high quantities in remote regions of the injured 164 heart at any point after injury. (scale bar $=100 \mu \mathrm{m} ;{ }^{*} \mathrm{p}<0.05,{ }^{* * *} \mathrm{p}<0.001$ relative to 165 uninjured). 


\subsection{Cardiomyocyte proliferation is limited following cryoinjury}

Proliferation of existing cardiomyocytes is a known driver of cardiac regeneration in zebrafish and neonatal mice (Chablais et al., 2011; Jopling et al., 2010; Porrello et al.; Schnabel et al., 2011). Staining for cardiomyocytes (cTnT) and phospho-histone H3 $(\mathrm{pH} 3)$ revealed low to absent levels of cardiomyocyte proliferation at every timepoint following injury (Fig. 5A,B), which at least partially accounts for a failure of cardiomyocytes to repopulate the injured area (Fig. 5A). However, proliferation of nonmyocytes was also quantified and revealed an increase in proliferative cells at 14 and $28 \mathrm{DPI}$ specifically in the injured region (Fig. 5A,C; $p<0.01$ at $14 \mathrm{DPI}$ and $p<0.001$ at $28 \mathrm{DPI}$ relative to uninjured fish; $p<0.01$ at $14 \mathrm{DPI}$ and $p<0.05$ at $28 \mathrm{DPI}$ relative to remote regions at that timepoint). This indicates that the injured region is being repopulated by non-myocytes following cryoinjury. This could potentially also be due to proliferation of dedifferentiated cells that fail to fully differentiate back into functioning cardiomyocytes.

\subsection{Grass carp exhibit persistent and chronic fibrosis following injury}

While fibrosis and cardiac regeneration are not mutually exclusive, scar resolution is necessary to allow infiltration of healthy tissue into the injured site. Masson's trichrome staining revealed that scar formation, visible as collagen-rich blue regions, started by 14 DPI and persisted through 56 DPI (Fig. 6A). These samples were stained with picrosirius red to assess the maturation and alignment of collagen fibers within the scar (Fig. 6B). Scars at $14 \mathrm{DPI}$ contained a large fraction of green fibers, indicative of immature collagens while scars at 28 and 56 DPI contained predominantly mature red fibers (Fig. 6B,C; mature fiber fraction $p<0.001$ between $14 \mathrm{DPI}$ and both 28 and 56 DPI). Collagen fibers in the scar were aligned along the ventricle wall at 14 DPI but became more randomly oriented by 56 DPI (Fig. 6B,D; $p<0.01$ between 14 and 56 DPI. This correlates with an increased presence of collagen fibers extending form the ventricle surface into the trabecular myocardium. Together these data indicate that the scar tissue is maturing over time with no evidence of regression within 8 weeks. 


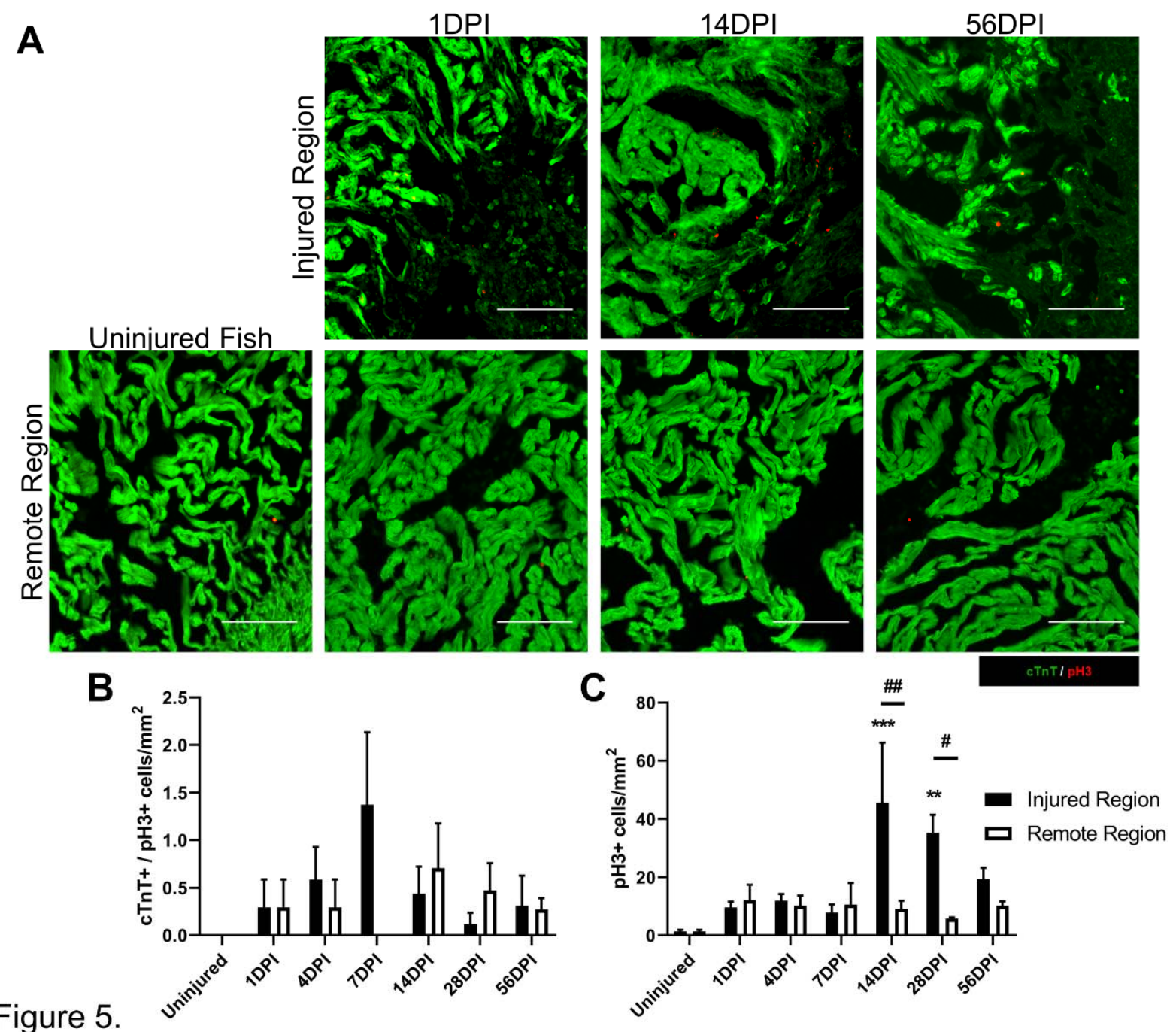

Figure 5.

197 Figure 5. Local proliferation of non-cardiomyocytes contributes to fibrosis.

198 (A) Co-staining of cTnT + cardiomyocytes and pH3+ cells reveals an increase in non-

199 myocyte proliferation at 14 DPI specifically in the injured zone. Quantification of

200 proliferating cell types reveals that (B) cardiomyocytes do not proliferate to repopulate

201 the injured region at any point after injury, but (C) a proliferating non-myocyte population

202 within the injured zone is present at 14 and $28 \mathrm{DPI}$ (scale bar $=100 \mu \mathrm{m} ;{ }^{* *} \mathrm{p}<0.01,{ }^{* * *} \mathrm{p}$

$203<0.001$ relative to uninjured; $\# p<0.05$, \#\#p $<0.01$ between regions). 
Figure 6.
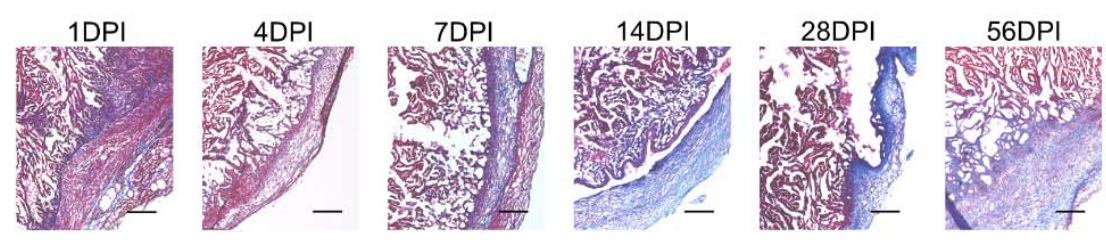

B

14DPI

28DPI

56DPI
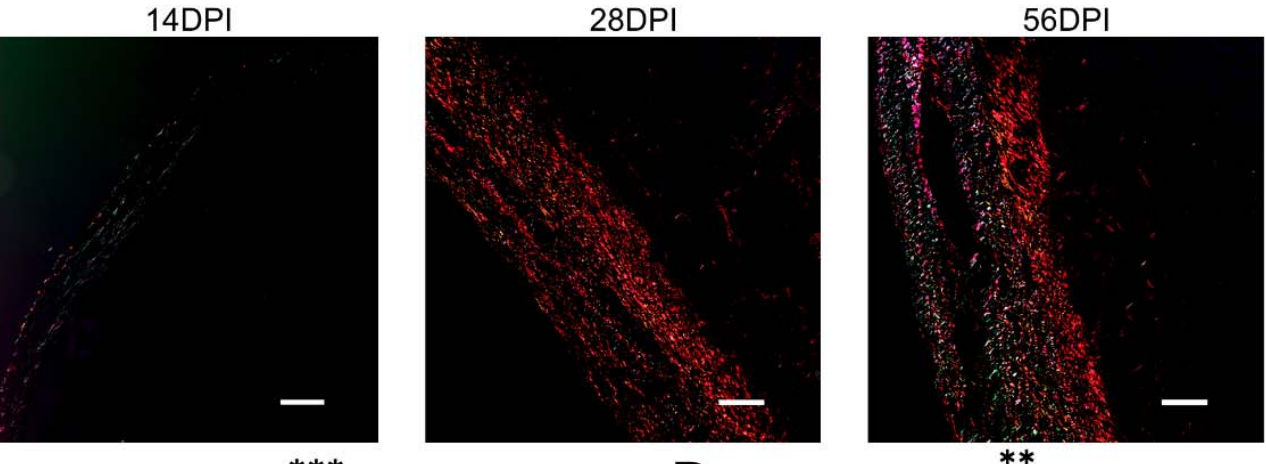

C

$* * *$

D
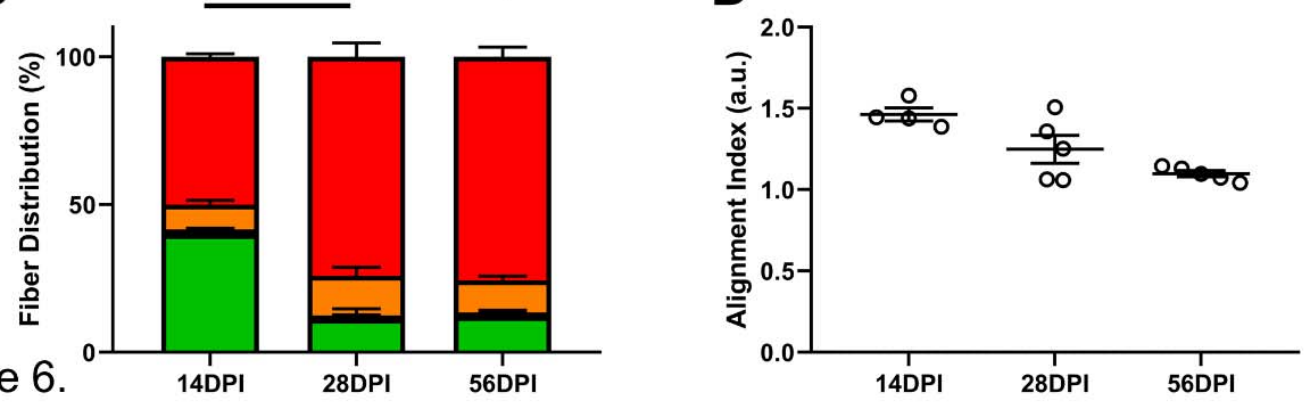

Figure 6: Scar formation and maturation in the ventricle following cryoinjury

(A) Masson's trichrome staining reveals the formation of a fibrotic scar in blue visible at $14,28$, and 56 DPI (scale bars $=200 \mu \mathrm{m})$.

(B) Picrosirius red staining of sections at the timepoints following scar formation show the presence of collagen fibers migrating into the intact myocardium (scale bars $=100 \mu \mathrm{m}$ ).

(C) Analysis of picrosirius red images by fiber color shows a shift from immature green fibers at 14 DPI to predominantly red

212 fibers at 28 and 56 DPI. (D) Collagen fibers become less aligned over time, resembling

213 a cross-hatch pattern in the injured region. $\left({ }^{* *} p<0.01,{ }^{* *} p<0.005\right.$ between groups). 
2.6 Fibroblasts depletion occurs across the ventricle following injury

216 Conventional wisdom on fibrosis in mammals and fish studied so far suggests

217 fibroblasts and activated myofibroblasts play a major role in ventricular remodeling,

218 depositing and restructuring the extracellular matrix (ECM) within the injured region.

219 Therefore, we quantified the density of vimentin + fibroblasts in both injured and remote

220 regions of grass carp hearts to assess their contribution to fibrosis. Unexpectedly,

221 fibroblast density decreased in both injured and remote areas of the heart following

222 cryoinjury (Fig. 7A). This depletion of vimentin + fibroblasts extended throughout the 8-

223 week study period in both injured and remote regions of the heart. Fibroblast density

224 was lowest at $4 \mathrm{DPI}$ in the injured region and $7 \mathrm{DPI}$ in remote regions of the heart (Fig.

$2257 \mathrm{C}$; injured region: $p<0.05$ at 1,7 , and $14 \mathrm{DPI}, \mathrm{p}<0.01$ at $4 \mathrm{DPI}$ relative to uninjured

226 fish; remote region: $p<0.05$ at 14,28 , and $56 \mathrm{DPI}, \mathrm{p}<0.01$ at 1 and $4 \mathrm{DPI}, \mathrm{p}<0.005$ at

$2277 \mathrm{DPI}$ relative to uninjured fish). Differences between injured and remote regions of the

228 heart at any single timepoint were non-significant, indicating this response is non-

229 localized. Surprisingly, a-smooth muscle actin (aSMA)+/vimentin+ myofibroblasts were

230 not found in any tissue analyzed in this study regardless of injury state or timepoint. The

231 injured zone contained aSMA+ cells at all timepoints beyond $1 \mathrm{DPI}$ in the injured

232 regions of the heart, but aSMA expression did not colocalize with vimentin. This

233 indicates that the myofibroblast phenotype may not be present in the grass carp or may

234 exist in a different state that does not include aSMA upregulation. Together this data

235 indicates widespread vimentin + fibroblast depletion in the grass carp heart following

236 injury and a lack of traditional myofibroblast differentiation. 
A

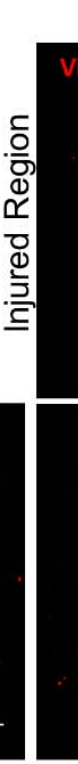

1DPl

14DPI

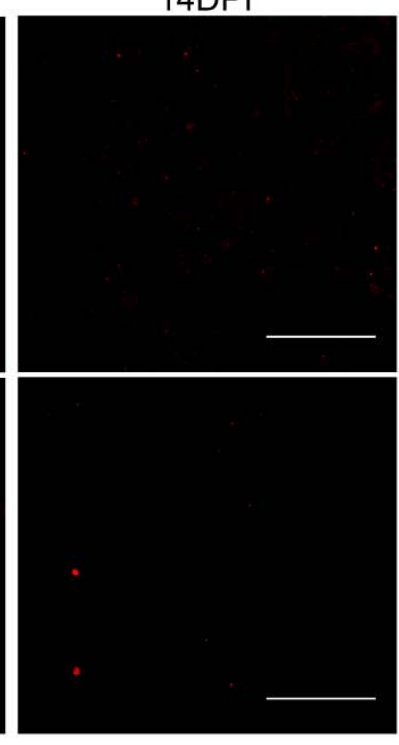

C 150

B

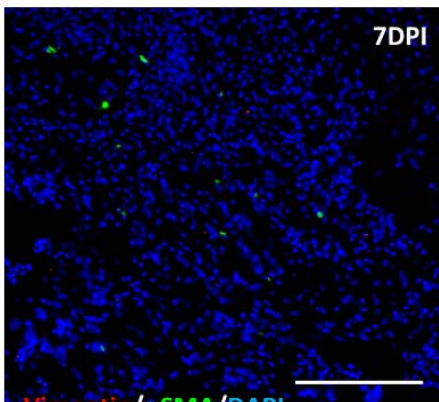

Figure 7.

Vimentin/aSMA/DAPI

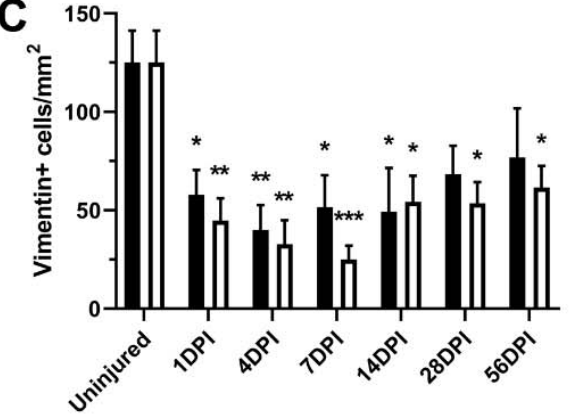

56DPI

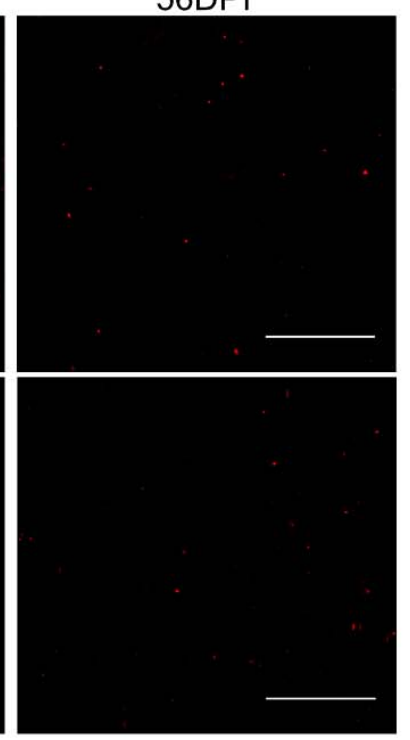

- Injured Region

口 Remote Region

239 Figure 7: Vimentin+ fibroblasts are depleted following injury.

240 (A) Representative images of vimentin+ fibroblasts are shown in both injured and

241 remote regions of the heart over $56 \mathrm{DPI}$. (B) aSMA+ cells were present at all timepoints

242 beyond $1 \mathrm{DPI}$, but no evidence was seen of vimentim+/aSMA+ co-expression,

243 indicating a lack of myofibroblast differentiation in this species following injury (scale bar

$244=100 \mu \mathrm{m}$ ). (C) Fibroblast density significantly decreases starting and 1 DPI does not

245 fully recover for the remainder of the study. No statistically significant differences are

246 present between injured and remote positions at any time point (scale bar $=100 \mu \mathrm{m} ;{ }^{*} \mathrm{p}$

$247<0.05,{ }^{* *} p<0.01,{ }^{* * *} p<0.001$ relative to uninjured fish). 


\subsection{Macrophage infiltration following cryoinjury}

Macrophages are known regulators of cardiac regeneration in zebrafish and fibrosis in non-regenerating species (Aurora et al., 2014; Frantz and Nahrendorf, 2014; Lai et al., 2017). Colony stimulating factor receptor $1+($ Csfr1+) macrophages were stained in both injured and remote regions of the heart following injury to determine their behavior in the grass carp (Chen et al., 2015). Csfr1+ cells were essentially nonexistent in any of the uninjured hearts analyzed. Immediately after injury, csfr1+ cells became more abundant, peaking at 14 DPI before returning to near-baseline levels at 28 and 56 DPI (Fig. 8; injured region: $p<0.05$ at $7 \mathrm{DPI}, \mathrm{p}<0.001$ at $14 \mathrm{DPI}$ relative to uninjured fish; remote region: $p<0.01$ at $7 \mathrm{DPI}, p<0.001$ at $14 \mathrm{DPI}$ relative to uninjured fish). It is worth noting that csfr1+ macrophages were still present in both injured and remote regions of the heart at $56 \mathrm{DPI}(n=5 / 5$ fish analyzed), although cell densities were not significantly different from baseline. Additionally, macrophage cell density was similar across injured and remote regions of the heart. This indicates that grass carp are neither exhibiting long-term chronic inflammation nor responding specifically to the injured region of the heart following cryoinjury.

\section{Discussion}

Coronary heart disease and its complications remain among the leading causes of death worldwide (Benjamin et al., 2018). Following an injury such as myocardial infarction, the human heart fails to regenerate. Instead, widespread fibrosis and chronic inflammation result in scar formation at the injury site and worsening cardiac function that eventually leads to heart failure (Frantz and Nahrendorf, 2014; Segura et al., 2014; Talman and Ruskoaho, 2016). Naturally, the phenomenon of heart regeneration has gained interest in order to better understand and prevent this adverse remodeling. Several animal models have been studied for this purpose (Cano-Martínez et al., 2010; Flink, 2002; Grivas et al., 2014; Lafontant et al., 2012a; Liao et al., 2017; Neff et al., 1996; Porrello et al., 2011; Poss et al., 2002; Stockdale et al., 2018). Among these models, zebrafish heart regeneration has been most extensively studied and has sparked interest in finding alternate models of cardiac disease in non-mammalian species to determine the driving mechanisms that dictate the cardiac injury response. 


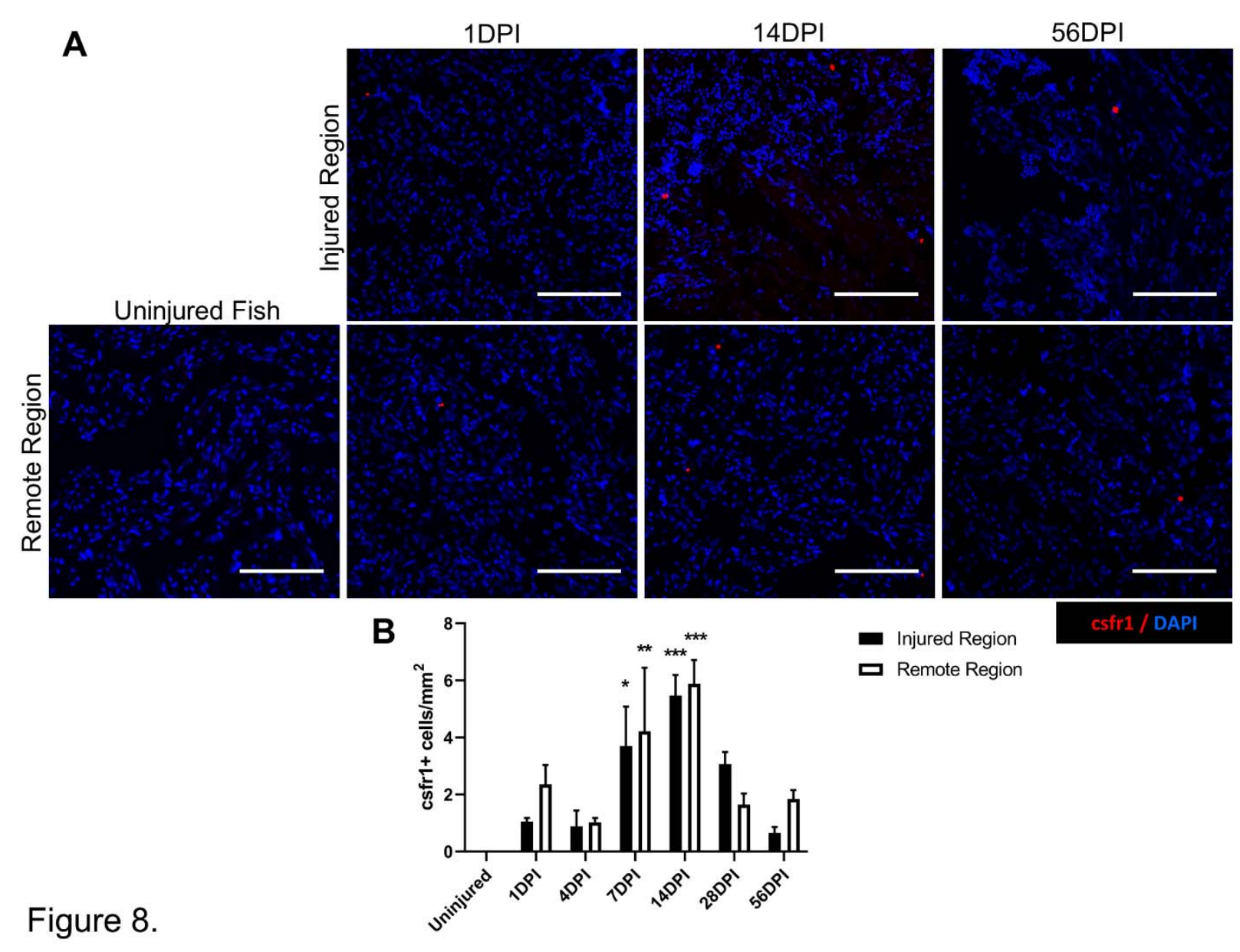

Figure 8: Macrophage populations show a non-localized response to cryoinjury.

282 (A) Representative images of csfr1 + macrophages are shown in both the injured regions 283 and regions of the heart far from the injured zone over time. (B) Quantification of csfr1+ 284 cell density reveals a peak in macrophage density at $14 \mathrm{DPI}$, although there is no 285 difference in density between injured and remote regions of the heart at any timepoint. 286 (scale bar $=100 \mu \mathrm{m} ;{ }^{*} p<0.05,{ }^{* *} p<0.01,{ }^{* * *} p<0.001$ relative to uninjured fish). 
Cardiac disease models in teleost fish have focused primarily on the Cypridinae family, which includes zebrafish (Poss et al., 2002), giant danio (Lafontant et al., 2012a), and goldfish (Grivas et al., 2014). Exploration of other fish families has revealed two species of fish that are incapable of cardiac regeneration, the medaka (Ito et al., 2014) and the Pachón variety of Astyanax mexicanus (Stockdale et al., 2018). Interestingly, these fish each exhibit unique rather than similar cellular responses to injury that result in chronic fibrosis. While comparisons between these fish and the widely studied zebrafish are possible, the evolutionary distance between these species limits the capabilities of comparative protein or RNA expression techniques (relation between investigated fish are shown in Fig. 1 (Yang et al., 2015)). Additional models of cardiac disease are needed to elucidate the commonalities among species that exhibit chronic fibrosis rather than regeneration. The present study reports the response of grass carp (Ctenopharyngodon idella) to cardiac cryoinjury. This is the first reported species within the Cyprinidae family that does not regenerate cardiac tissue after injury. It is also the first large fish species reported in the context of cardiac injury to our knowledge. Instead of widespread cardiomyocyte proliferation and transient fibrosis seen in zebrafish, giant danio, and goldfish (Grivas et al., 2014; Lafontant et al., 2012a; Poss et al., 2002), the grass carp exhibits very limited if any cardiomyocyte proliferation, chronic fibrosis, and decreased cardiac function up to 8 weeks post-injury.

Development of a cardiac injury model in large fish requires additional considerations not relevant to small fish species. The bony operculum in the grass carp restricts access to the ventricle, making resection of the apex impossible, even with the use of retractors. For that reason, a cryoinjury model was developed in which a $2 \mathrm{~mm}$ stainless steel probe was applied to the ventricle wall to create a necrotic injured area. This model is not as widely used as resection, but it more closely mimics the initial fibrosis and necrosis seen in human heart disease (Chablais et al., 2011). Unlike small fish species which can be immobilized on a moistened sponge for the short duration of heart injury, the long period of anesthesia required to complete this procedure requires the use of a recirculating pump to allow tricaine anesthetic and water flow across the gills. A recirculation pump system was able to maintain consistent surgical depth of anesthesia with successful recovery from surgery. Additionally, the use of florfenicol antibiotic is a 
necessary precaution during the injury procedure to prevent the onset of bacterial infection at the incision site, which is not necessary in small fish. Omission of florfenicol resulted in widespread infection and ulceration with 14 days of surgery. We can't exclude the possibility that florfenicol or Melafix treatments interfere with the carps' response to cardiac injury as their effect on regeneration has not been documented. Development of this model ultimately resulted in $>95 \%$ survival within 14 days of injury and $>90 \%$ survival to the final 56-day timepoint with consistent injury size that damaged both the compact and trabecular myocardium. High levels of apoptosis were seen as early as $1 \mathrm{DPI}$ in the injured region, which resolved quickly to baseline levels of apoptosis (Fig. 4). This demonstrates the ability of the cryoprobe to create a local and acute injury to the heart that results in an area of tissue necrosis similar to zebrafish and neonatal mouse models (Chablais et al., 2011; Darehzereshki et al., 2015; Polizzotti et al., 2015).

Proliferation of cardiomyocytes is a known driver of cardiac regeneration in nonmammalian species and neonatal mammals (Cano-Martínez et al., 2010; Flink, 2002; Gonzalez-Rosa et al., 2011; Lai et al., 2017; Liao et al., 2017; Porrello et al., 2011; Sallin et al., 2015; Schnabel et al., 2011; Stockdale et al., 2018; Vargas-González et al., 2005; Yu et al., 2018); further, a regenerative phenotype has not yet been reported in the absence of cardiomyocyte proliferation (Ito et al., 2014; Lin and Pu, 2014; Marshall et al., 2017). However, the recent finding of widespread cardiomyocyte proliferation in non-regenerative Pachón Astyanax mexicanus suggests that this behavior is not the sole factor that dictates a regenerative response (Stockdale et al., 2018). In the present study, widespread cardiomyocyte proliferation in grass carp was not observed, similar to the response of medaka and adult mammals after injury (Ito et al., 2014; Lin and Pu, 2014).

Proliferation of non-myocytes varies among non-regenerative species, and their underlying functions in the context of cardiac wound healing are currently unclear. Grass carp exhibit an increase in non-myocyte proliferation that peaks at $14 \mathrm{DPI}$ specifically in the injured region (Fig. 4). Interestingly, the non-regenerative Pachón Astyanax mexicanus exhibits a similar peak in non-myocyte proliferation at this timepoint, but only in remote regions of the heart rather than the injured zone (Stockdale 
et al., 2018). Non-regenerative Xenopus laevis do not exhibit increased cell proliferation at all (Marshall et al., 2017). Localized proliferation of non-myocytes has thus far only been described in zebrafish and axolotl; however, this occurs at much earlier stages, peaking 1 week post-injury (Chablais et al., 2011; Godwin et al., 2017; Gonzalez-Rosa et al., 2011; Sánchez-Iranzo et al., 2018; Schnabel et al., 2011; Xu et al., 2018; Yu et al., 2018). Taken together, these results indicate that the response of grass carp cardiomyocytes closely resemble non-regenerative medaka and adult mammals, but the proliferation of non-myocytes is a delayed onset of behavior seen in zebrafish during cardiac regeneration. The identification of proliferating non-myocytes in these species has not been fully elucidated but could be key to understanding the species-level differences in cardiac injury response.

Fibrosis occurs in both regenerative and non-regenerative species after cryoinjury due to the presence of necrotic tissue (Chablais et al., 2011; Lai et al., 2017; Marshall et al., 2017; Mizutani et al., 2015; Sánchez-Iranzo et al., 2018). For cardiomyocytes to replace the scar tissue, fibrosis must be transient rather than chronic. Masson's trichrome staining indicated that scar formation is present in grass carp up to $56 \mathrm{DPI}$; further, picrosirius red staining revealed that the collagen fibers become more mature and penetrate the trabecular myocardium by $56 \mathrm{DPI}$, showing no signs of scar regression (Fig. 6). This response is similar to the medaka, where scar formation is seen at 14 DPI (Ito et al., 2014), and Xenopus laevis, where scar formation is seen by 1 month postinjury (Marshall et al., 2017). Collagen fibers in zebrafish, however, are visible by 7 DPI, and the scarred region is completely surrounded by healthy tissue by 21 DPI (Chablais et al., 2011). From this comparison, the progression of fibrosis appears similar to the non-regenerative medaka and Xenopus laevis.

Surprisingly, grass carp exhibit chronic fibrosis despite a depletion of fibroblasts in the ventricle following injury (Fig. 7). Fibroblasts and myofibroblasts are typically the largest contributors of matrix deposition following injury (Ivey and Tallquist, 2016; Souders et al., 2009) in both regenerative and non-regenerative models. More specifically, fibroblast density in the heart increases within 1 week of injury in every reported species of cardiac injury, including zebrafish (Gonzalez-Rosa et al., 2011; Sánchez-Iranzo et al., 2018; Xu et al., 2018), medaka (Ito et al., 2014), neonatal mice (Mizutani et al., 2015), 
381

382

383

384

385

386

387

388

389

390

391

392

393

394

395

396

397

398

399

400

401

402

403

404

405

406

407

408

409

410

411

and adult mammals (Talman and Ruskoaho, 2016). Instead, grass carp see a marked reduction in fibroblasts in the injury zone and beyond that persists across the entire length of the study (Fig. 7). Regardless, scar formation and chronic fibrosis are the outcome in this disease model. These seemingly contradictory results are unique to this species and indicate other cell types are primarily responsible for secreting fibrotic ECM within the injured region. In support of this theory, zebrafish endocardial cells are known to secrete collagen within the injury region 7 DPI (Sánchez-Iranzo et al., 2018), although their ultimate contribution to scar formation is limited relative to fibroblasts. Endocardial cells may be playing a role in grass carp scar formation as well, but we were unfortunately unable to assess the cell type contributing to this fibrotic behavior due to availability of species-specific reagents. Future studies could identify the role of endocardial cells in scar formation and maintenance in the injured grass carp heart. Another surprising result in this species is the lack of aSMA+/vimentin+ myofibroblasts (Fig. 7B). Other studies have used aSMA as a putative marker of myofibroblasts and shown infiltration of these cells to the injured region (Ito et al., 2014; Yu et al., 2018), but the lack of colocalization shown in this study indicate that aSMA alone is not sufficient to identify myofibroblasts in fish. Our data indicates that myofibroblasts, if they exist in the grass carp, could have a different expression profile than mammals, making the colocalization of aSMA and vimentin an ineffective probe for myofibroblast differentiation.

The immune response has repeatedly been shown as a vital component of regeneration. In zebrafish, depletion of macrophages impedes regeneration (Lai et al., 2017), while stimulation of macrophages in the non-regenerative medaka promotes cardiomyocyte proliferation and scar resolution (Lai et al., 2017). Grass carp exhibit a delayed and muted csfr1+ macrophage response, peaking at $14 \mathrm{DPI}$; in contrast, macrophage infiltration peaks between 1 and 4 DPI in zebrafish and Xenopus tropicalis and reach baseline by 14-16 DPI (De Preux Charles et al., 2016; Liao et al., 2017; Xu et al., 2018). Macrophage presence in the grass carp does not mimic the chronic response seen in mammals either. Adult mice exhibit a macrophage peak near 5 DPI (Talman and Ruskoaho, 2016) and remain above baseline for at least 8 weeks (Sager et al., 2016) while grass carp reach near-baseline levels by 28 DPI. Surprisingly, 
412 macrophages are not restricted to the injured region, and an organ-wide response is

413 seen instead (Fig. 8). A previous study in the zebrafish and medaka suggests that early

414 macrophage response is essential to a regenerative phenotype, so this finding at least

415 partially explains the lack of regeneration seen in the grass carp (Lai et al., 2017).

416 However, the effect of a localized versus organ-wide immune response has not yet

417 been explored and warrants further investigation.

418 Due to the novelty of this model in the context of cardiac disease, caveats of using the 419 grass carp as a study model must be acknowledged. The size of these fish relative to 420 other fish models imposes restrictions on the housing and throughput capabilities in a 421 laboratory setting. The injury protocol takes longer in grass carp than in zebrafish and 422 has the potential to cause higher levels of stress. A previous study in zebrafish has 423 examined the detrimental effects of stress on cardiac regeneration (Sallin and 424 Jaźwińska, 2016), and stress may have exasperated the fibrotic response seen in the 425 present study. Due to the sparsity of grass carp studies performed, molecular 426 techniques and protein characterization in this species is not as well-developed as other 427 models of cardiac disease such as mice and zebrafish. This limits the ability to identify 428 specific cell types or proteins in the heart. However, with recent publication of the grass carp genome (Wang et al., 2015) and widespread availability of total protein and gene expression techniques, this limitation can be addressed in future studies.

431 The present study, in addition to identifying a new potential model of cardiac disease, 432 describes a surgical procedure for cardiac injury adaptable to other large species of fish. 433 Although there are essentially two phenotypes to cardiac injury, our evidence in the 434 grass carp along with the contributions of others suggest that a fibrotic rather than a 435 regenerative response can be due to multiple different changes in cell behavior. While 436 Pachón Astyanax mexicanus, grass carp, and medaka all fail to regenerate, multiple 437 differences in cell behavior exist between these species that each lead to similar fibrotic 438 phenotypes. The grass carp provides a model to study fibrosis apparently independent 439 of fibroblast infiltration. Future studies in these animals could identify cell types and 440 proteins previously unknown to contribute to chronic fibrosis in the context of cardiac 441 disease. Studying these models has implications not only to understanding the 
442 underlying mechanisms of regeneration and chronic fibrosis but also in identifying 443 potential targets to treat human heart disease.

\section{4. Materials and Methods}

\subsection{Animal Procurement and Care}

All work with live animals was approved by the Cornell University Institutional Animal Care and Use Committee prior to starting experiments. Results from a total of 35 fish are presented in this study. Live grass carp weighing $435 \pm 112 \mathrm{~g}$ were purchased from a local fish farm (Candor, NY, USA) (Fig. 1B). Fish were housed in indoor 360 gallon capacity tanks measuring roughly $1.2 \times 1.2 \times 0.9$ meters with at least once full water turnover a day. Water temperature was kept between 18 and $20^{\circ} \mathrm{C}$. Nets and tarps over the tanks prevented fish from jumping out of the tank, a natural defense mechanism in this species. A maximum of 6 fish were housed per tank. Fish were fed commercial trout pellets several times per day. Any fish showing signs of fin rot were isolated and treated with 7-day soaks in aquarium salt (1.5 tsp/gal) and a 7-day treatment with Melafix (API Fishcare, Chalfont, PA, USA) according to package instructions.

\subsection{Cryoinjury procedure}

To induce anesthesia, carp were captured individually and submerged in a bath of tricaine methanesulfonate (MS-222) and sodium bicarbonate (both at $150 \mathrm{mg} / \mathrm{L}$, tricaine supplied by Western Chemical Inc., Ferndale, WA, USA) to induce anesthesia. Surgical depth anesthesia was observed by slowed or stopped opercula movements, ceased

462 body movements, lack of response to a pinch at the base of the tail, and floating in a supine position. A recirculating water pump was then fitted to the fish's mouth to continuously flush a tricaine and sodium bicarbonate solution (each at $100 \mathrm{mg} / \mathrm{L}$ ) over

465 the gills for the duration of surgery. After placing the fish in a supine position, excess mucus was removed from the underside with gauze and the surgical surface was cleaned twice with povidone-iodine applied in the direction of scale growth. Pre-surgical analgesia of lidocaine (Henry Schein Animal Health, Dublin, OH, USA) was administered subcutaneously (1 mg/kg), and carprofen (Putney Inc., Portland, ME,

470 USA) was administered IM $(2.5 \mathrm{mg} / \mathrm{kg})$; a dose of florfenicol (30 mg $/ \mathrm{kg}$; Merck Animal

471 Health, Summit, NJ, USA) was administered IM to prevent bacterial infection due to 472 surgery. 
473 Three to four rows of scales directly overlying the heart were removed by pulling 474 caudally until they released. The exposed skin was washed twice with povidone-iodine solution. The heart was exposed through a $1.5 \mathrm{~cm}$ incision along the length of the fish,

476 cutting through the skin, underlying musculature, and pericardial sac. Alm retractors (17008-07; Fine Science Tools Inc., Foster City, CA, USA) were used to separate the operculum approximately 5-8 $\mathrm{mm}$, allowing direct visualization of the ventricle (Fig. 1C). Excess blood and moisture were removed from the ventricle surface and body cavity with cotton-tipped swabs. A liquid nitrogen-cooled stainless steel probe measuring 2 $\mathrm{mm}$ in diameter was then directly applied to the ventricle for 30 seconds along the free wall as close to the apex as possible to create the cryoinjury. After 30 seconds, a few drops of saline were used to thaw the probe and tissue, allowing trauma-free removal of the probe. A successful injury is easily observed due to an instantaneous lack of ventricle movement that occurs upon application of the cryoprobe and recovers quickly after thawing. Additionally, the injured region is seen as a bright red mark on the ventricle surface after removing the cryoprobe. Excess moisture and blood were removed from the body cavity and ventricle surface before removing the retractors. The incision was closed using 4-0 polyglyconate suture in a simple interrupted pattern, closing the muscle and skin layers separately whenever possible. Immediately after wound closure, the fish was moved to a recovery tank and fresh water moved constantly over its gills until opercular movements and swimming behavior recovered.

493 Following recovery, the fish was returned to its original tank.

495 Immediately before sacrifice, fish were anesthetized in a tricaine/sodium bicarbonate 496 bath (each at 150mg/L) and then maintained at 100mg/L. A Vevo 2100 series 497 ultrasound fitted with a MS400 transducer (FUJIFILM VisualSonics Inc., Toronto, ON, 498 Canada) was used to obtain long-axis images of the heart in at least 3 separate 499 locations. Short-axis imaging was not possible due to interference by the operculum. 500 Immediately after imaging, the fish was placed in a high concentration tricaine/sodium 501 bicarbonate bath (each at 300mg/L) for euthanasia.

502 FAC, a functional measurement of cardiac function, was calculated by manually 503 outlining and measuring the area occupied by the ventricle at systole and diastole. FAC 
504 percentage was then calculated as $\frac{\text { diastolic area-systolic area }}{\text { diastolic area }} \times 100$. FS, another

505 measurement of ventricular contractility, was determined by measuring the distance

506 from base to apex at systole and diastole. Similarly, FS, expressed as a percentage,

507 was calculated as $\frac{\text { diastolic length-systolic length }}{\text { diastolic length }} \times 100$. At least 3 separate heart cycles

508 were analyzed for FAC and FS measurements. Echocardiography-based

509 measurements were made using ImageJ (NIH, Bethesda, MD, USA). Decreases in

510 either of these measurements are indicative of a loss in cardiac function, and both are

511 used clinically to assess heart function after myocardial infarction (MI).

5124.4 Tissue processing and histology

513 Immediately after euthanasia, the entire heart was extracted, including the bulbous

514 arteriosus and atria. The atrium was often destroyed during processing and is not

515 always present in histology samples. Hearts were fixed for 1 hour in $4 \%(\mathrm{w} / \mathrm{v})$

516 paraformaldehyde, cryoprotected overnight in $30 \%(\mathrm{w} / \mathrm{v})$ sucrose, embedded in O.C.T.

517 compound, and frozen in dry ice-cooled isopentane. Long-axis serial sections were

518 collected at a thickness of $8 \mu \mathrm{m}$ with each section separated by roughly $400 \mu \mathrm{m}$ on the 519 slide.

520 AFOG staining was performed as previously described (Darehzereshki et al., 2015;

521 Poss et al., 2002) to mark healthy tissue, fibrin, and collagen. For each of these stains,

522 injury area was calculated as percentage of the ventricle area covered by collagen or

523 fibrin-stained tissue. This was repeated for each tissue section, and the largest relative

524 injured area was taken as the injury size for that heart.

525 Masson's trichrome staining was performed to indicate which timepoints following injury

526 were characterized by collagen deposition and therefore appropriate for picrosirius red

527 staining. Picrosirius red staining was then performed on these samples to assess the

528 maturity and alignment of collagen fibers within the ventricle after injury under polarized

529 light microscopy. Within the injured region of each heart, the distribution of green,

530 yellow, orange, and red fibers were calculated using MATLAB as previously described

531 (Rich and Whittaker, 2005). Immature collagen fibers were defined as green and yellow

532 fibers, and mature fibers were defined as orange and red. The percentage of mature

533 fibers in the injured region was used for statistical comparisons, although data for all 
534 defined colors are presented. Alignment was calculated as previously described (Long 535 et al., 2017) using a fast Fourier transform (FFT) macro in ImageJ, where index values

536 close to 1 indicate random fiber alignment and higher index values indicate increased

537 fiber alignment.

$538 \quad 4.5$ Apoptosis in the heart following injury

539 To assess levels of apoptosis in carp hearts following cryoinjury, the Click-iTTM Plus

540 TUNEL assay (Invitrogen, Carlsbad, CA, USA) was used according to the

541 manufacturer's instructions. Prior to staining, tissue sections were fixed with $4 \%$

542 formaldehyde for 15 minutes followed by permeabilization in $0.25 \%(v / v)$ triton $x-100$ for

54315 minutes. The percentage of apoptotic cells was assessed in both the injured region

544 and an uninjured region of the heart far from the injury (remote) to determine the role of

545 both local and systemic cell death in the tissue response.

$546 \quad 4.6$ Immunofluorescent staining and imaging

547 Immunofluorescent staining of tissue sections was used to determine the behavior of

548 different cell populations following cryoinjury. All immunofluorescent stains were

549 performed after 5 -minute fixation in $2 \%(\mathrm{w} / \mathrm{v})$ formaldehyde, permeabilizing for ten

550 minutes in $0.2 \%(\mathrm{v} / \mathrm{v})$ triton $\mathrm{x}-100$, and blocking for 1 hour in $5 \%$ normal goat serum at

551 room temperature. Primary antibodies were incubated in $1 \%$ normal goat serum at $4^{\circ} \mathrm{C}$

552 overnight and detected with fluorescent secondary antibodies (A-11032, A-11037, A-

553 11029, ThermoFisher Scientific, 1:500 dilution) through a 1-hour incubation at 37으. All

554 brightfield and immunofluorescent imaging was performed on a Nikon Eclipse Ti2-E

555 inverted microscope, and image analysis performed with NIS Elements software unless

556 otherwise specified (Tokyo, Japan).

557 Proliferation of cardiomyocytes is a known mechanism of cardiac regeneration in

558 zebrafish (Chablais et al., 2011; Jopling et al., 2010; Schnabel et al., 2011). Therefore,

559 the presence of actively proliferating cardiomyocytes was assessed by co-staining of

560 cardiac troponin T (cTnT; MA5-12960, ThermoFisher Scientific, 1:40 dilution) found in

561 cardiomyocytes and pH3 (ab5176, Abcam, 1:200 dilution), a mitotic marker. The density

562 of $\mathrm{pH} 3+$ cardiomyocytes and $\mathrm{pH} 3+$ non-myocytes were calculated at each timepoint

563 within the injured region and a remote region of the ventricle. 
564 Fibroblasts and myofibroblasts are responsible for secreting and remodeling the 565 extracellular matrix (ECM) following cardiac injury (Ma et al., 2017). The density of vimentin+ cells (ab8069, Abcam, 1:100 dilution) in injured and remote regions were

567 quantified over time, and myofibroblasts, defined as vim+/aSMA+, (aSMA, ab8211,

568 Abcam, 1:100 dilution) were identified to detect a potential pro-fibrotic response.

569 Immune cell response following injury is known to play an active role in cardiac

570 regeneration in other species (Lai et al., 2017; Xu et al., 2018). Therefore, csfr1+

571 (GTX128677, Genetex, 1:200 dilution) macrophage density was assessed by

572 immunofluorescent staining of both injured and remote regions of the grass carp

573 ventricle following injury to detect both the location and time-dependence of

574 macrophage infiltration into the damaged tissue.

$575 \quad 4.7$ Data and statistical analysis

576 All data is presented as mean \pm SEM, and $n=3-5$ fish per timepoint were used for

577 each measurement. Statistically significant differences in means were detected using

578 independent analysis of variance (ANOVA); one-way independent ANOVA was used

579 when a single measurement was made per animal, and two-way independent ANOVA

580 was used when comparisons were made across time and between remote and injured

581 regions of the heart. Tukey's post hoc testing was used for AFOG injury size,

582 proliferation of non-myocytes, and picrosirius red fiber color distribution and alignment in

583 order to compare across all timepoints and regions. Sidak's post hoc test was used to

584 compare FAC, FS, TUNEL, fibroblast density, and csfr1+ macrophage density

585 measurements relative to uninjured fish samples.

\section{5. Acknowledgements}

587 We thank Dr. Kenneth Poss and Dr. Voot Yin for providing their AFOG protocols and

588 the entire veterinary and animal care staff at Cornell University for their assistance

589 throughout this study. Additionally, we thank Dr. Helene Marquis for assisting with our

590 fish anesthesia systems and monitoring.

591 6. Competing Interests

592 The authors have no competing interests to disclose.

\section{7. Funding}

594 This study is supported by a startup fund from Cornell University 
Aurora, A. B., Porrello, E. R., Tan, W., Mahmoud, A. I., Hill, J. A., Bassel-Duby, R., Sadek, H. A. and Olson, E. N. (2014). Macrophages are required for neonatal heart regeneration. J. Clin. Invest. 124, 1382-92.

Benjamin, E. J., Salim Virani, C. S., Chair Clifton Callaway, C.-V. W., Chamberlain, A. M., Alexander Chang, M. R., Susan Cheng, M., Stephanie Chiuve, M. E., Mary Cushman, S., Francesca Delling, F. N., Rajat Deo, M., et al. (2018). Heart Disease and Stroke Statistics-2018 Update A Report From the American Heart Association. Circulation 137, 67-492.

Cano-Martínez, A., Vargas-González, A., Guarner-Lans, V., Prado-Zayago, E., León-Olea, M. and Nieto-Lima, B. (2010). Functional and structural regeneration in the axolotl heart (Ambystoma mexicanum) after partial ventricular amputation. Arch. Cardiol. Mex.

Chablais, F., Veit, J., Rainer, G. and Jawiska, A. (2011). The zebrafish heart regenerates after cryoinjury-induced myocardial infarction. BMC Dev. Biol.

Chen, Q., Lu, X.-J. and Chen, J. (2015). Identification and functional characterization of the CSF1R gene from grass carp Ctenopharyngodon idellus and its use as a marker of monocytes/macrophages. Fish Shellfish Immunol. 45, 386-398.

Darehzereshki, A., Rubin, N., Gamba, L., Kim, J., Fraser, J., Huang, Y., Billings, J., Mohammadzadeh, R., Wood, J., Warburton, D., et al. (2015). Differential regenerative capacity of neonatal mouse hearts after cryoinjury. Dev. Biol.

De Preux Charles, A. S., Bise, T., Baier, F., Marro, J. and Jaźwińska, A. (2016). Distinct effects of inflammation on preconditioning and regeneration of the adult zebrafish heart. Open Biol.

Ding, L., Kuhne, W. W., Hinton, D. E., Song, J. and Dynan, W. S. (2010). Quantifiable biomarkers of normal aging in the Japanese Medaka fish (Oryzias latipes). PLoS One.

Flink, I. L. (2002). Cell cycle reentry of ventricular and atrial cardiomyocytes and cells 
within the epicardium following amputation of the ventricular apex in the axolotl, Amblystoma mexicanum: Confocal microscopic immunofluorescent image analysis of bromodeoxyuridine-label. Anat. Embryol. (Berl).

Frantz, S. and Nahrendorf, M. (2014). Cardiac macrophages and their role in ischaemic heart disease. Cardiovasc. Res.

Godwin, J. W., Debuque, R., Salimova, E. and Rosenthal, N. A. (2017). Heart regeneration in the salamander relies on macrophage-mediated control of fibroblast activation and the extracellular landscape. NPJ Regen. Med. 2,.

Gómez-Requeni, P., Conceição, L. E. C., Jordal, A. E. O. and Rønnestad, I. (2010). A reference growth curve for nutritional experiments in zebrafish (Danio rerio) and changes in whole body proteome during development. Fish Physiol. Biochem.

Gonzalez-Rosa, J. M., Martin, V., Peralta, M., Torres, M. and Mercader, N. (2011). Extensive scar formation and regression during heart regeneration after cryoinjury in zebrafish. Development.

Grivas, J., Haag, M., Johnson, A., Manalo, T., Roell, J., Das, T. L., Brown, E., Burns, A. R. and Lafontant, P. J. (2014). Cardiac repair and regenerative potential in the goldfish (Carassius auratus) heart. Comp. Biochem. Physiol. C. Toxicol. Pharmacol. 163, 14-23.

Ito, K., Morioka, M., Kimura, S., Tasaki, M., Inohaya, K. and Kudo, A. (2014). Differential reparative phenotypes between zebrafish and medaka after cardiac injury. Dev. Dyn.

Ivey, M. J. and Tallquist, M. D. (2016). Defining the Cardiac Fibroblast. Circ. J.

Jopling, C., Sleep, E., Raya, M., Martí, M., Raya, A. and Belmonte, J. C. I. (2010). Zebrafish heart regeneration occurs by cardiomyocyte dedifferentiation and proliferation. Nature.

Lafontant, P. J., Burns, A. R., Grivas, J. A., Lesch, M. A., Lala, T. D., Reuter, S. P., Field, L. J. and Frounfelter, T. D. (2012a). The Giant Danio (D. Aequipinnatus) as A Model of Cardiac Remodeling and Regeneration. Anat. Rec. 
Lafontant, P. J., Burns, A. R., Grivas, J. A., Lesch, M. A., Lala, T. D., Reuter, S. P., Field, L. J. and Frounfelter, T. D. (2012b). The giant danio (D. aequipinnatus) as a model of cardiac remodeling and regeneration. Anat. Rec. (Hoboken). 295, 23448.

Lai, S.-L., Marín-Juez, R., Moura, P. L., Kuenne, C., Lai, J. K. H., Tsedeke, A. T., Guenther, S., Looso, M. and Stainier, D. Y. (2017). Reciprocal analyses in zebrafish and medaka reveal that harnessing the immune response promotes cardiac regeneration. Elife.

Lenhoff, S. G. and Lenhoff, H. M. (1986). Hydra and the Birth of Experimental Biology, 1744: Abraham Trembley's Memoires Concerning the Polyps. Pacific Grove, CA: Boxwood Press.

Liao, S., Dong, W., Lv, L., Guo, H., Yang, J., Zhao, H., Huang, R., Yuan, Z., Chen, Y., Feng, S., et al. (2017). Heart regeneration in adult Xenopus tropicalis after apical resection. Cell Biosci.

Lin, Z. and Pu, W. T. (2014). Strategies for cardiac regeneration and repair. Sci. Transl. Med.

Long, D. W., Johnson, N. R., Jeffries, E. M., Hara, H. and Wang, Y. (2017).

Controlled delivery of platelet-derived proteins enhances porcine wound healing. $J$. Control. Release.

Ma, Y., lyer, R. P., Jung, M., Czubryt, M. P. and Lindsey, M. L. (2017). Cardiac Fibroblast Activation Post-Myocardial Infarction: Current Knowledge Gaps. Trends Pharmacol. Sci.

Marshall, L., Vivien, C., Girardot, F., Péricard, L., Demeneix, B. A., Coen, L. and Chai, N. (2017). Persistent fibrosis, hypertrophy and sarcomere disorganisation after endoscopyguided heart resection in adult Xenopus. PLoS One.

Michalopoulos, G. K. (2007). Liver Regeneration. J. Cell. Physiol. 213, 286.

Mizutani, M., Wu, J. C. and Nusse, R. (2015). Fibrosis of the neonatal mouse heart after cryoinjury is accompanied by Wnt signaling activation and epicardial-to- 
mesenchymal transition. J. Am. Heart Assoc.

680

Morgan, T. H. (1901). Regeneration. Columbia University Biological Series, Vol. VII. Vol. VII. New York: The Macmillan company.

Neff, A. W., Dent, A. E. and Armstrong, J. B. (1996). Heart development and regeneration in urodeles. Int. J. Dev. Biol. 40, 719-25.

Polizzotti, B. D., Ganapathy, B., Walsh, S., Choudhury, S., Ammanamanchi, N., Bennett, D. G., dos Remedios, C. G., Haubner, B. J., Penninger, J. M. and Kühn, B. (2015). Neuregulin stimulation of cardiomyocyte regeneration in mice and human myocardium reveals a therapeutic window. Sci. Transl. Med. 7, 281 ra45.

Porrello, E. R., Mahmoud, A. I., Simpson, E., Johnson, B. A., Grinsfelder, D., Canseco, D., Mammen, P. P., Rothermel, B. A., Olson, E. N. and Sadek, H. A. Regulation of neonatal and adult mammalian heart regeneration by the miR-15 family.

Porrello, E. R., Mahmoud, A. I., Simpson, E., Hill, J. A., Richardson, J. A., Olson, E. N. and Sadek, H. A. (2011). Transient Regenerative Potential of the Neonatal Mouse Heart. Science (80-. ). 331, 1078-1080.

Poss, K. D., Wilson, L. G. and Keating, M. T. (2002). Heart regeneration in zebrafish. Science 298, 2188-90.

Rich, L. and Whittaker, P. (2005). Collagen and Picrosirius Red Staining $\square$ : a Polarized Light Assessment of Fibrillar Hue and Spatial Distribution. Brazilian J. Morphol. Sci.

Sager, H. B., Hulsmans, M., Lavine, K. J., Moreira, M. B., Heidt, T., Courties, G., Sun, Y., Iwamoto, Y., Tricot, B., Khan, O. F., et al. (2016). Proliferation and Recruitment Contribute to Myocardial Macrophage Expansion in Chronic Heart Failure. Circ. Res.

Sallin, P. and Jaźwińska, A. (2016). Acute stress is detrimental to heart regeneration in zebrafish. Open Biol.

Sallin, P., de Preux Charles, A. S., Duruz, V., Pfefferli, C. and Jaźwińska, A. (2015). 
A dual epimorphic and compensatory mode of heart regeneration in zebrafish. Dev. Biol.

Sánchez-Iranzo, H., Galardi-Castilla, M., Sanz-Morejón, A., González-Rosa, J. M., Costa, R., Ernst, A., Sainz de Aja, J., Langa, X. and Mercader, N. (2018). Transient fibrosis resolves via fibroblast inactivation in the regenerating zebrafish heart. Proc. Natl. Acad. Sci.

Schnabel, K., Wu, C. C., Kurth, T. and Weidinger, G. (2011). Regeneration of cryoinjury induced necrotic heart lesions in zebrafish is associated with epicardial activation and cardiomyocyte proliferation. PLoS One.

Segura, A., Frazier, O. and Buja, L. (2014). Fibrosis and heart failure. Hear. Fail Rev 19, 173-185.

Souders, C. A., Bowers, S. L. K. and Baudino, T. A. (2009). Cardiac fibroblast: The renaissance cell. Circ. Res.

Stich, D. S., Dicenzo, V., Frimpong, E. A., Jiao, Y. and Murphy, B. R. (2013). Growth and Population Size of Grass Carp Incrementally Stocked for Hydrilla Control. North Am. J. Fish. Manag. 33, 14-25.

Stockdale, W. T., Lemieux, M. E., Killen, A. C., Zhao, J., Hu, Z., Riepsaame, J., Hamilton, N., Kudoh, T., Riley, P. R., van Aerle, R., et al. (2018). Heart Regeneration in the Mexican Cavefish. Cell Rep.

Talman, V. and Ruskoaho, H. (2016). Cardiac fibrosis in myocardial infarction-from repair and remodeling to regeneration. Cell Tissue Res.

Underwood, J. L., Hestand, R. S. and Thompson, B. Z. (1986). Gonad Regeneration in Grass Carp Following Bilateral Gonadectomy. Progress. Fish-Culturist 48, 5456.

Vargas-González, A., Prado-Zayago, E., León-Olea, M., Guarner-Lans, V. and Cano-Martínez, A. (2005). Myocardial regeneration in Ambystoma mexicanum after surgical injury. Arch. Cardiol. Mex. 
Wang, Y., Lu, Y., Zhang, Y., Ning, Z., Li, Y., Zhao, Q., Lu, H., Huang, R., Xia, X., Feng, Q., et al. (2015). The draft genome of the grass carp (Ctenopharyngodon idellus) provides insights into its evolution and vegetarian adaptation. Nat. Genet.

Xu, S., Webb, S. E., Lau, T. C. K. and Cheng, S. H. (2018). Matrix metalloproteinases (MMPs) mediate leukocyte recruitment during the inflammatory phase of zebrafish heart regeneration. Sci. Rep.

Yang, L., Sado, T., Vincent Hirt, M., Pasco-Viel, E., Arunachalam, M., Li, J., Wang, X., Freyhof, J., Saitoh, K., Simons, A. M., et al. (2015). Phylogeny and polyploidy: Resolving the classification of cyprinine fishes (Teleostei: Cypriniformes). Mol. Phylogenet. Evol. 85, 97-116.

Ye, L., D’Agostino, G., Loo, S. J., Wang, C. X., Su, L. P., Tan, S. H., Tee, G. Z., Pua, C. J., Pena, E. M., Cheng, R. B., et al. (2018). Early Regenerative Capacity in the Porcine Heart. Circulation CIRCULATIONAHA.117.031542.

Yu, J. K., Sarathchandra, P., Chester, A., Yacoub, M., Brand, T. and Butcher, J. T. (2018). Cardiac regeneration following cryoinjury in the adult zebrafish targets a maturation-specific biomechanical remodeling program. Sci. Rep. 
A

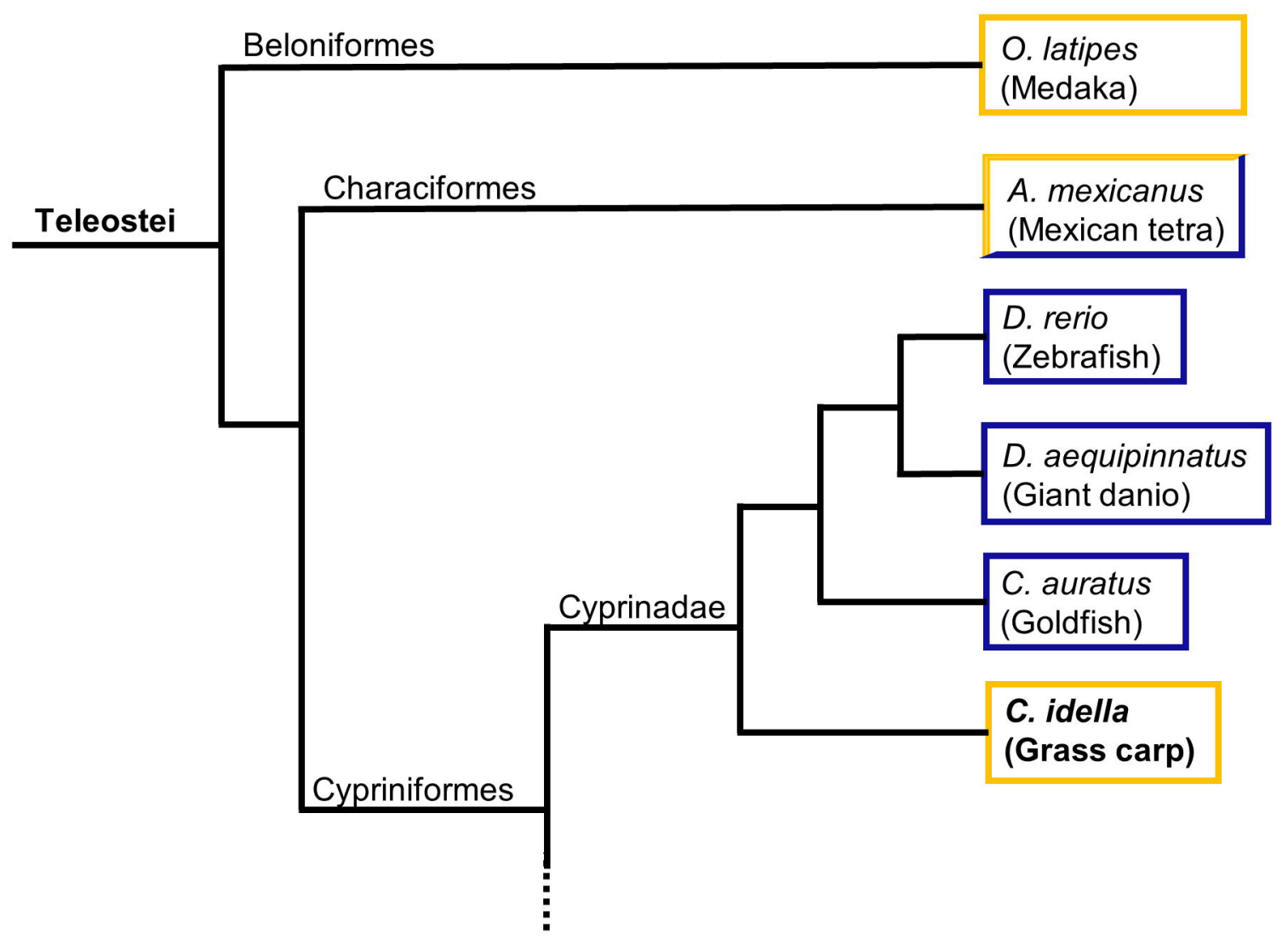

B

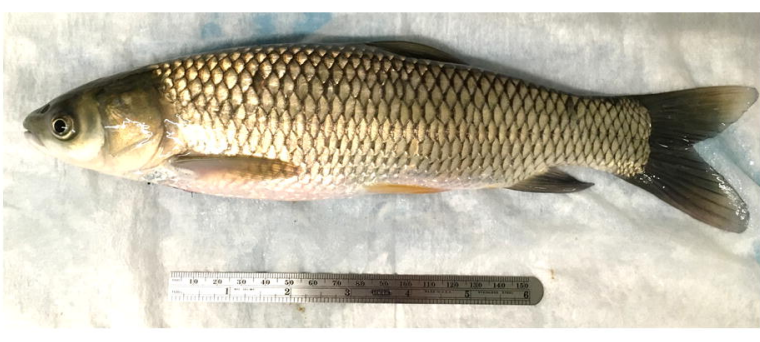

C

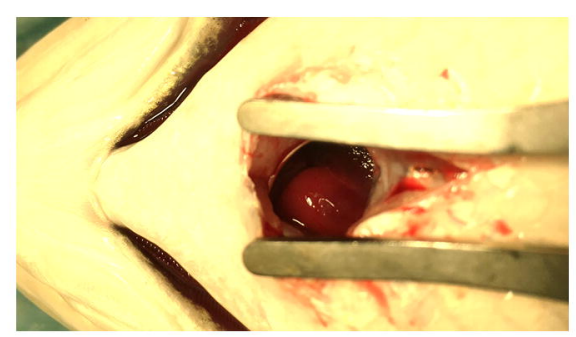

Regeneration

Fibrosis

D

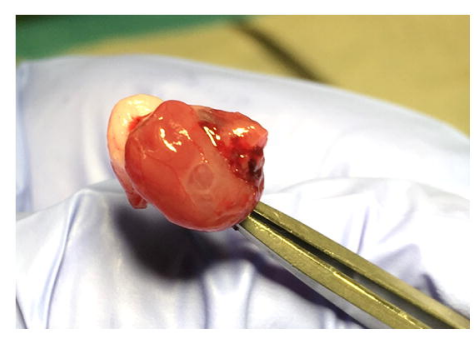

\section{Figure 1.}



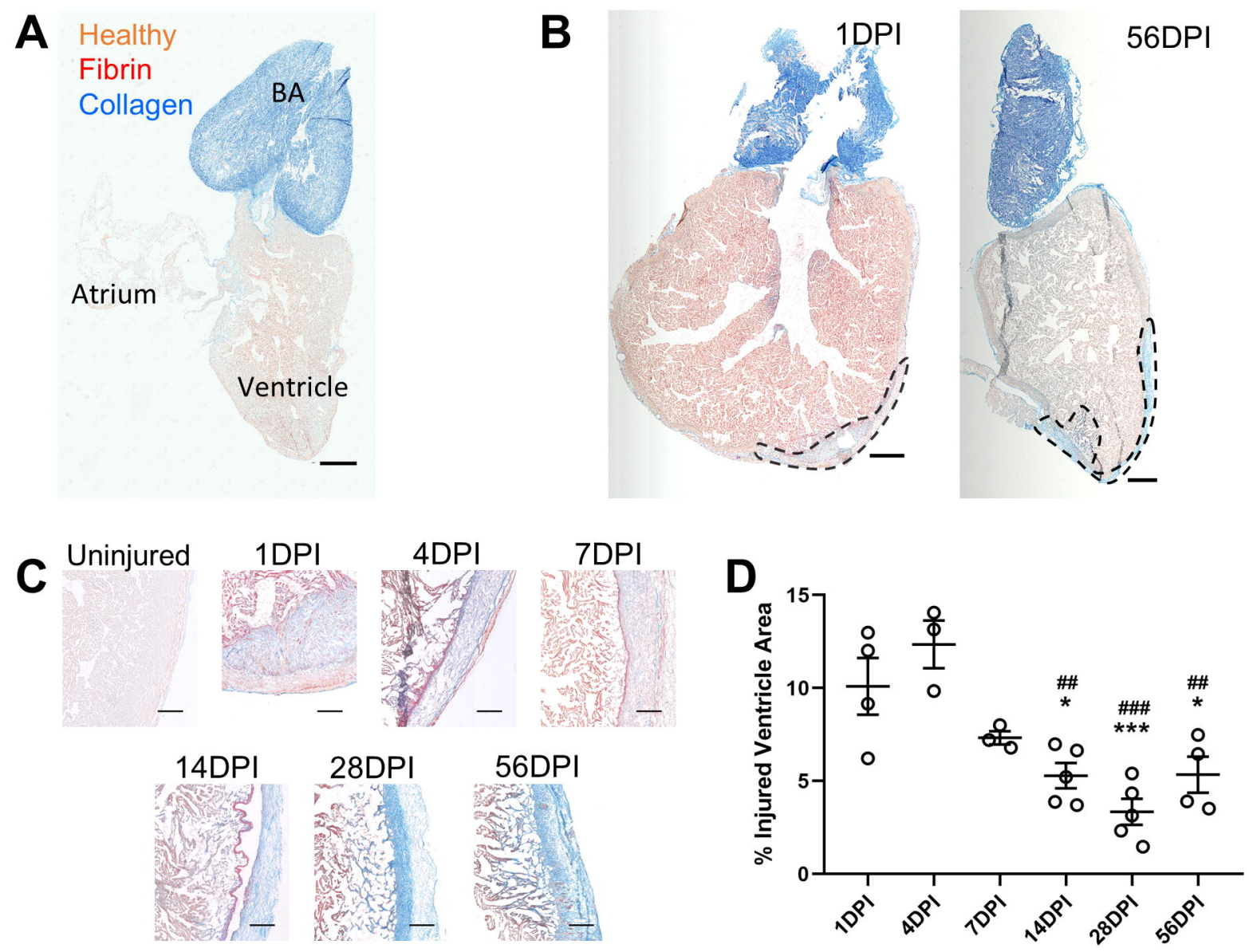

Figure 2. 
A

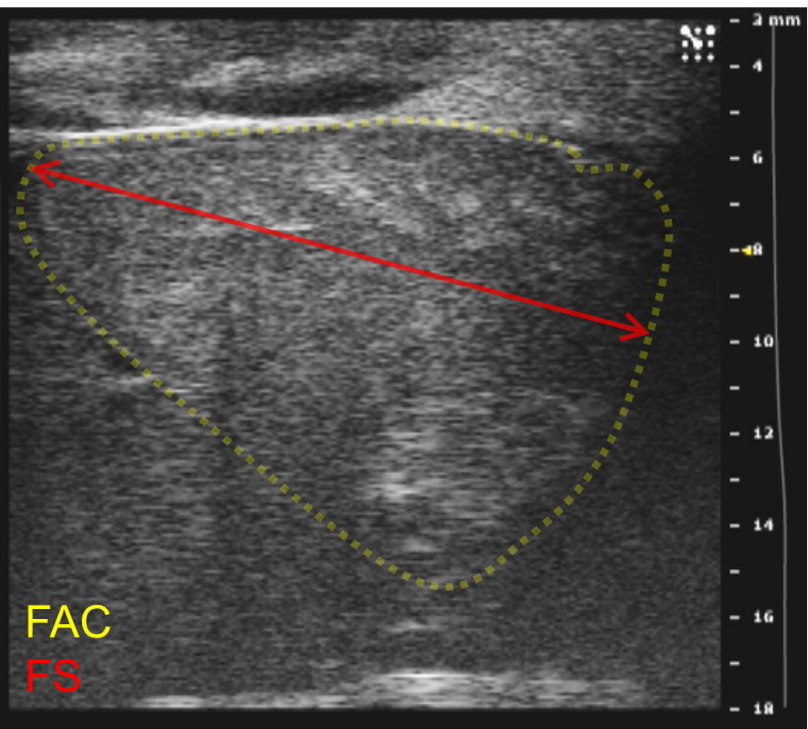

B

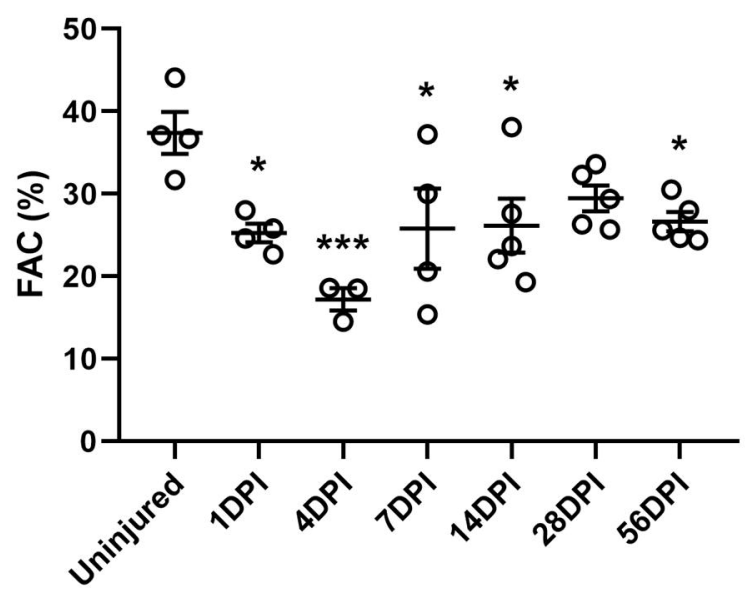

Systole

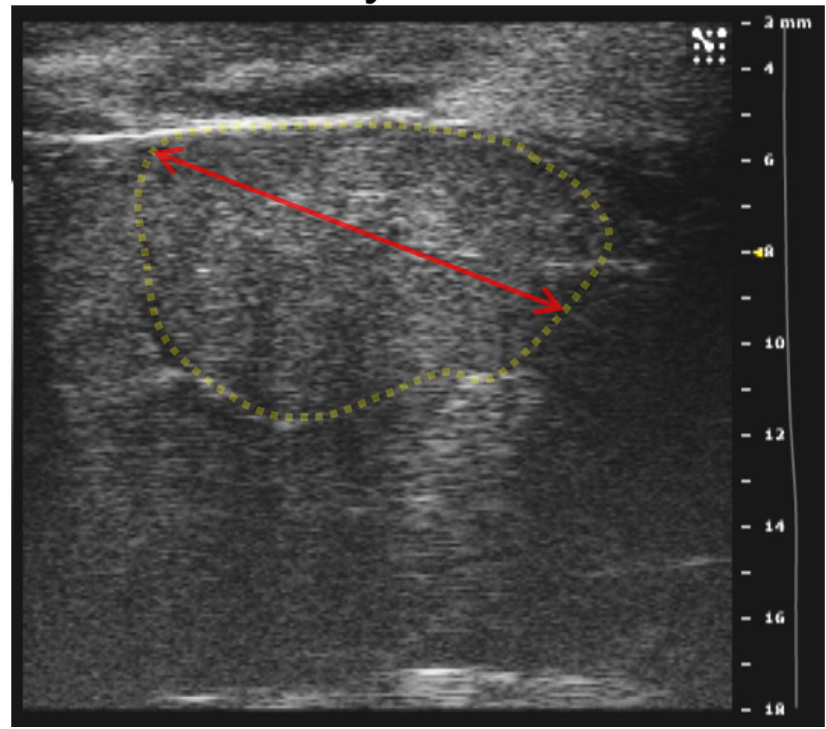

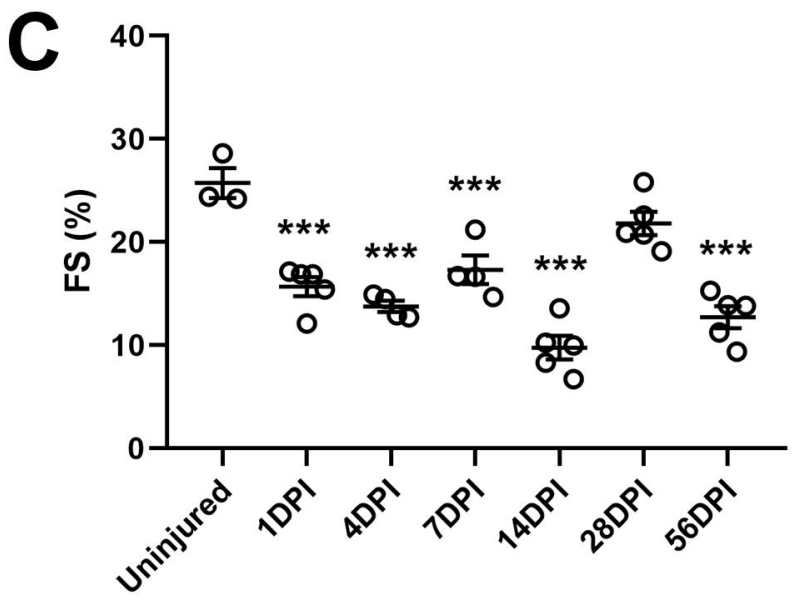

Figure 3. 
A Uninjured
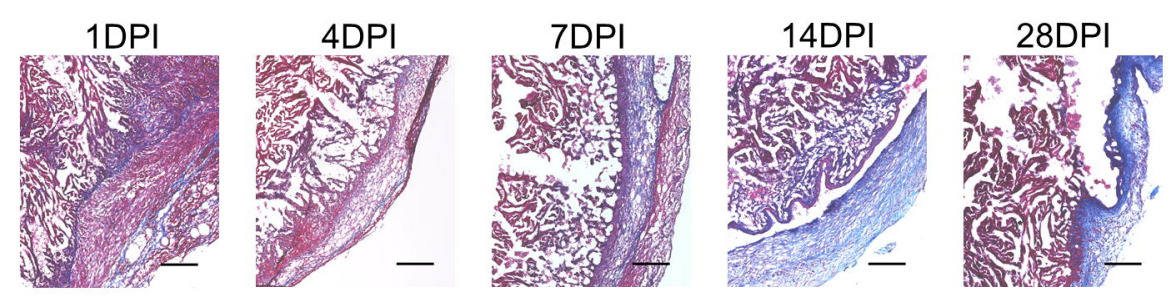

56DPI

B

14DPI

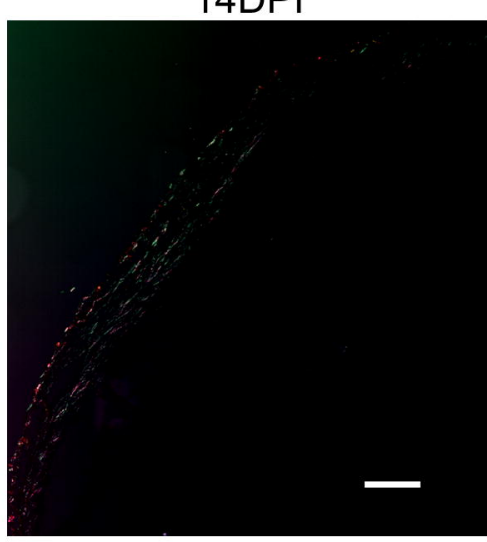

28DPI

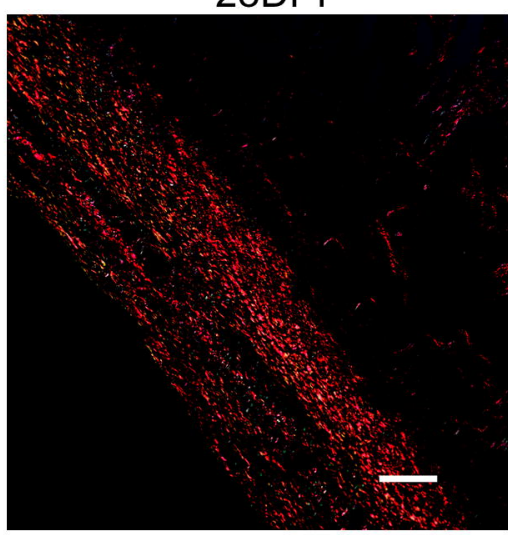

C

***

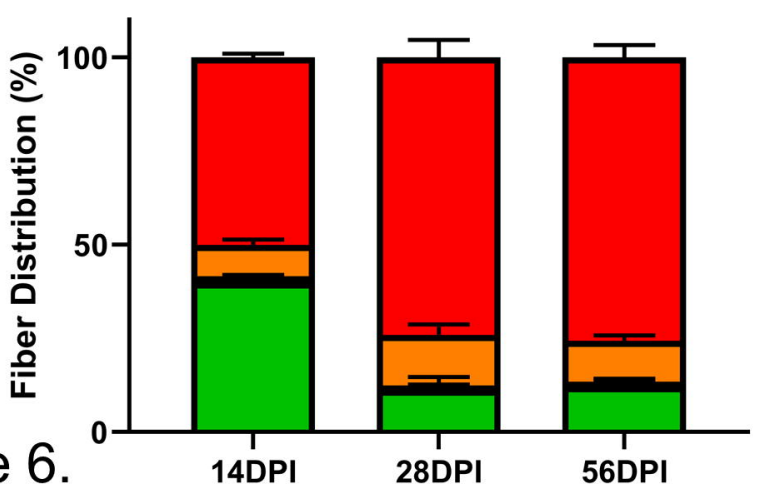

D

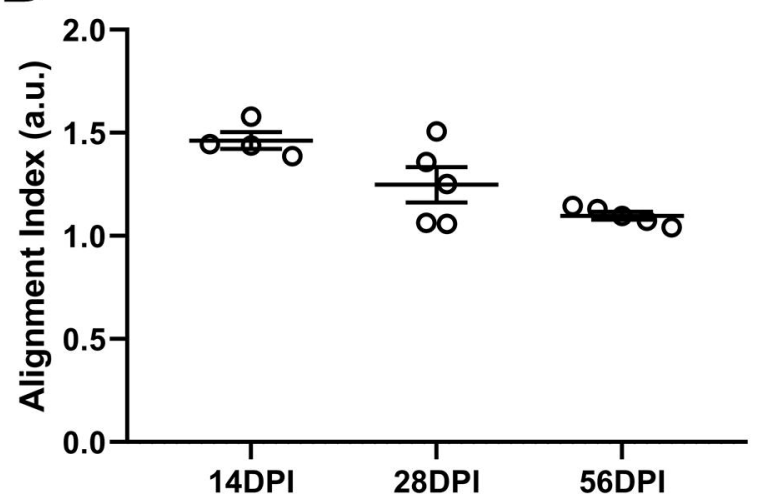

Figure 6.

14DPI

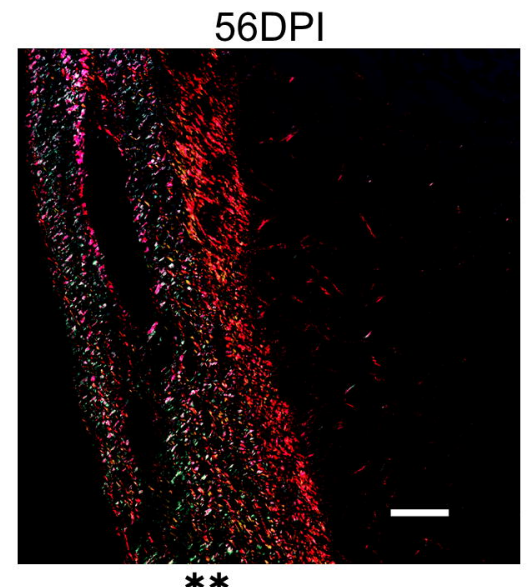


A

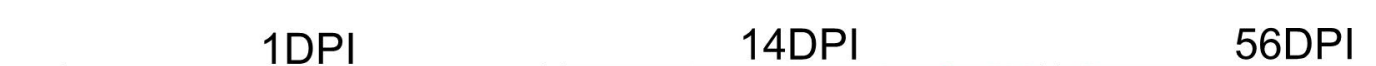

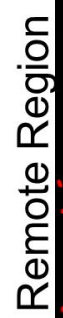

Uninjured Fish

Figure 7. Vimentin/aSMA/DAPI

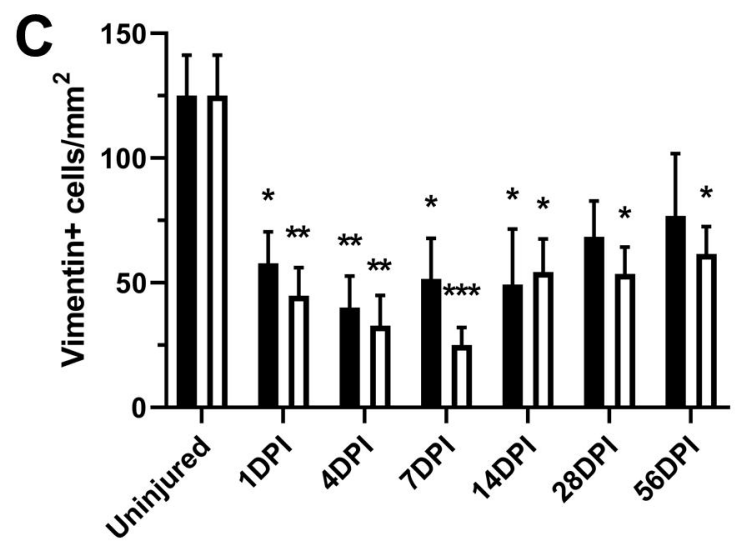

- Injured Region

ㅁ Remote Region 
